\title{
The metalloproteinase-proteoglycans ADAMTS7 and ADAMTS12 provide an innate, tendon-specific protective mechanism against heterotopic ossification
}

Timothy J. Mead, ${ }^{1}$ Daniel R. McCulloch, ${ }^{1}$ Jason C. Ho, ${ }^{1,2}$ Yaoyao Du, ${ }^{1}$ Sheila M. Adams, ${ }^{3}$ David E. Birk, ${ }^{3}$ and Suneel S. Apte ${ }^{1}$

'Department of Biomedical Engineering and the Orthopaedic and Rheumatologic Institute, Cleveland Clinic Lerner Research Institute, Cleveland, Ohio, USA. ${ }^{2}$ Department of Orthopaedic Surgery and the Orthopaedic and Rheumatology Institute, Cleveland Clinic, Cleveland, Ohio, USA. ${ }^{3}$ Departments of Molecular Pharmacology and Physiology and Orthopaedics and Sports Medicine, University of South Florida, Morsani College of Medicine, Tampa, Florida, USA.

Heterotopic ossification $(\mathrm{HO})$ is a significant clinical problem with incompletely resolved mechanisms. Here, the secreted metalloproteinases ADAMTS7 and ADAMTS12 are shown to comprise a unique proteoglycan class that protects against a tendency toward $\mathrm{HO}$ in mouse hindlimb tendons, menisci, and ligaments. Adamts7 and Adamts12 mRNAs were sparsely expressed in murine forelimbs but strongly coexpressed in hindlimb tendons, skeletal muscle, ligaments, and meniscal fibrocartilage. Adamts $7^{-/-}$Adamts $12^{-/-}$mice, but not corresponding single-gene mutants, which demonstrated compensatory upregulation of the intact homolog mRNA, developed progressive $\mathrm{HO}$ in these tissues after 4 months of age. Adamts7 ${ }^{-/-}$Adamts $12^{-/-}$ tendons had abnormal collagen fibrils, accompanied by reduced levels of the small leucine-rich proteoglycans (SLRPs) biglycan, fibromodulin, and decorin, which regulate collagen fibrillogenesis. $\mathrm{Bgn}^{-/ 0} \mathrm{Fmod}^{-/-}$mice are known to have a strikingly similar hindlimb $\mathrm{HO}$ to that of Adamts7 ${ }^{-1-}$ Adamts12-/- mice, implicating fibromodulin and biglycan reduction as a likely mechanism underlying $\mathrm{HO}$ in Adamts7 $7^{-/-}$Adamts12-/- mice. Interestingly, degenerated human biceps tendons had reduced ADAMTS7 mRNA compared with healthy biceps tendons, which expressed both ADAMTS7 and ADAMTS12. These results suggest that ADAMTS7 and ADAMTS12 drive an innate pathway protective against hindlimb $\mathrm{HO}$ in mice and may be essential for human tendon health.

Authorship note: Daniel McCulloch and Yaoyao Du are retired.

Conflict of interest: The authors have declared that no conflict of interest exists.

Submitted: January 19, 2017 Accepted: February 28, 2018 Published: April 5, 2018

Reference information: JCI Insight. 2018;3(7):e92941. https:// doi.org/10.1172/jci.insight.92941.

\section{Introduction}

Secreted or cell-surface proteases that cleave extracellular matrix (ECM) components have significant roles in connective tissue formation, remodeling, and disease (1-3). The ADAMTS proteinases are a class of 19 secreted metalloenzymes with major roles in assembly and degradation of ECM molecules, many of which were revealed by human genetic disorders and mouse genetic models $(4,5)$. For example, ADAMTS2 is essential for procollagen amino-propeptide processing and the absence of this enzyme results in severe skin fragility in Ehlers-Danlos syndrome (dermatosparaxis type) (6). ADAMTS4 and ADAMTS5 mediate aggrecan proteolysis, a major mechanism of cartilage degradation in osteoarthritis (7-9). The WeillMarchesani syndrome spectrum arising from ADAMTS10 and ADAMTS17 mutations has connected these proteinases to the assembly of fibrillin microfibrils (reviewed in refs. 1,10 ). However, the functions of many ADAMTS proteases in connective tissue biology and disease remain unknown.

ADAMTS7 and ADAMTS12 constitute a clade of this family related to a single gene in the early chordate Ciona intestinalis, suggesting an origin by duplication of an ancestral gene $(11,12)$. A rare protein-coding ADAMTS7 variant leading to impaired ADAMTS7 zymogen processing by furin was linked to human coronary artery disease, and reduced atherogenesis was seen in Adamts7-null mice (13-15). ADAMTS7 was also found to mediate vascular wall remodeling and repair (16). ADAMTS7 and ADAMTS12 were 
causally implicated in cartilage destruction in arthritis via proteolysis of cartilage oligomeric protein (COMP) and in endochondral ossification using in vitro approaches (17-20). In contrast to the involvement of ADAMTS7 in coronary artery disease, unequivocal evidence for specific functions in musculoskeletal tissues using genetically engineered mutants is presently unavailable. Other than reduced atherogenesis, Adamts7-null mutants were reportedly normal, and Adamts12-null mice were reported to be developmentally normal, albeit with enhanced susceptibility to tumors $(14,21)$.

ADAMTS7 and ADAMTS12 have all the structural hallmarks of ADAMTS proteases, such as multiple thrombospondin type 1 repeats (TSRs), but unlike other family members, each contains a large mucinlike module containing putative glycan attachment sites located between 2 TSR clusters (12). Attachment of the glycosaminoglycan (GAG) chondroitin sulfate (CS) within the ADAMTS7 mucin-like module was previously demonstrated (12), but whether comparable modification of ADAMTS12 occurs is not known. Since proteoglycans (PGs) are crucial for musculoskeletal function and collagen fibrillogenesis (22-24), definitive investigation of ADAMTS12 as a prospective PG is relevant to its functional analysis.

Homologous ADAMTS proteases were previously shown to cooperate functionally by the appearance of highly penetrant phenotypes in combined null mutants (reviewed in ref. 25). Upon establishing that ADAMTS7 and ADAMTS12 constituted a unique class of metalloproteinase-PGs whose mRNAs are coexpressed in tendons, muscle, and menisci, mice with combined Adamts 7 and Adamts12 inactivation were generated for investigation of musculoskeletal functions. The findings establish a cooperative role for ADAMTS7 and ADAMTS12 in regulating tendon collagen fibril architecture and as innate inhibitors of HO that are relevant to human tendon degeneration.

\section{Results}

GAG attachment is an evolutionary hallmark of ADAMTS7 and ADAMTS12. GAG modification of ADAMTS12 was investigated to ask whether it constituted a distinct ADAMTS subfamily with ADAMTS7, which was previously shown to be a CS PG. The ADAMTS7 and ADAMTS12 mucin modules lie within identical domain structures (Figure 1, A and B), and like their C. intestinalis ortholog (UniProt ID F6Q5T5, Figure 1C), contain Ser-Gly or Gly-Ser motifs with adjacent or nearby acidic residues. Such dipeptides constitute typical GAG attachment sites, with the GAG chain being attached to the Ser residue (26). PGs may be secreted as a mixture of unmodified and modified core protein and electrophoresis of sulfated PGs typically results in a smear or retention in the stacking gel. Analysis of the medium of FLAG-tagged ADAMTS12-expressing cells showed 2 distinct anti-FLAG reactive bands at approximately 300 and $350 \mathrm{kDa}$, as well as a smear of higher molecular weights (Figure 1D; see complete unedited blots in the supplemental material). Treatment of ADAMTS12-conditioned medium with chondroitinase ABC, which degrades CS/dermatan sulfate (DS) GAGs but not with keratanase I (targeting keratan sulfate GAG) + II or heparitinases I + III (targeting heparan sulfate GAG) prior to electrophoresis led to increased intensity of anti-FLAG (ADAMTS12) reactive bands larger than $250 \mathrm{kDa}$ (Figure 1D; see complete unedited blots in the supplemental material), suggesting that ADAMTS12 was a CS/DS PG. The increased band intensity upon chondroitinase ABC treatment, moreover, suggested that a significant proportion of CS/DS-modified ADAMTS12 was of higher molecular mass or retained in the stacking gel without chondroitinase ABC treatment. That ADAMTS12 did not migrate as a sharp band of the predicted molecular mass $(177 \mathrm{kDa})$ even after treatment with these enzymes likely reflects extensive $O$-glycosylation and $N$-glycosylation within the mucin module (Figure 1B) (12). Ser ${ }^{157}$ and Ser $^{1159}$ were mutated to Ala (these Ser residues are in Ser-Gly dipeptides within strongly acidic contexts) and the mutants were analyzed alongside wild-type ADAMTS12 by Western blotting using anti-FLAG antibody and monoclonal antibody $(\mathrm{mAb}) 2035$ after treatment with or without chondroitinase $\mathrm{ABC}$. $\mathrm{mAb}$ 2035 exclusively recognizes CS stubs with an unsaturated glucuronic acid terminus derived from chondroitin 6-sulfate after chondroitinase ABC digestion and does not cross-react with keratan or chondroitin 4-sulfate. These stubs are absent in native, i.e., undigested CS or DS PGs and in molecules that lack CS. Wild-type ADAMTS12 and the Ser ${ }^{1157}$ Ala mutant migrated as 2 bands of approximately 300 and $350 \mathrm{kDa}$ without prior chondroitinase $\mathrm{ABC}$ digestion, whereas digestion with chondroitinase $\mathrm{ABC}$ increased the signal intensity of these bands and generated an additional band of approximately $375 \mathrm{kDa}$ (Figure 1E; see complete unedited blots in the supplemental material). In contrast, migration of the $\mathrm{Ser}^{157+1159} \mathrm{Ala}$ mutant was unaltered by chondroitinase $\mathrm{ABC}$ digestion (Figure 1E; see complete unedited blots in the supplemental material). mAb 2035 detected CS stubs after chondroitinase ABC treatment of wild-type ADAMTS12 and Ser ${ }^{157}$ Ala ADAMTS12 (Figure 1E; see complete unedited blots in the supplemental material). mAb 2035 reactivity was present, 
A

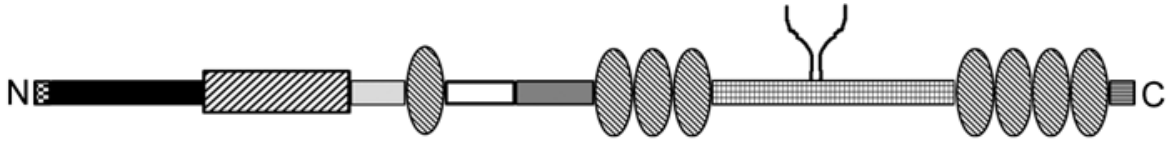

国signal peptide catalytic domain $\square$ disintegrin-like domain $\square$ spacer region / CS chains

Drodomain TSR

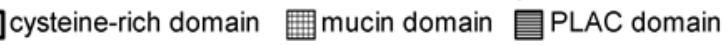

B

\section{TSR4}

Human SVSCGGGVRI RSVTCAKNHDEPCDVTRKPNSRALCGLQQCPSSRRVLKPNKGTISNGKNP 1016 Mouse SVSCGGGVRI RSVTCAKNLNEPCDKTRKPNSRALCGLQQCPF SRRVLKPNKDIAP SGKNQ 1020 $* * * * * * * * * * * * * * * * *: ; * * * * * * * * * * * * * * * * * * * * * * * * * * *, \ldots * * *$

Human PT-----LKPVPPPTSRPRMLTTPTGPESMSTSTPAISSPSPTTASKEGDLGGKQWQDSS 1071

Mouse STAEHDPFKP I PAPTSRPTPLSTPTVPESMSTSTPTINSLGSTIASQE-DANGMGWQNNS 1079 $.^{*} \quad:^{* *}:{ }^{*}, * * * * *^{*}: *^{* *} * * * * * * * * *:^{*},{ }^{*} \ldots *^{* * *}: *^{*} .^{*}{ }^{* * *}: .^{*}$

Human TQPE LSSRYL ISTGSTSQPILTSQSLSIQPSEENVSSSDTGPTSEGGLVATTTSGSGLSS 1131

Mouse TQAEEGSHF PTSSGSTS QVPVTSWSLSIQ PDDENVSSSA I GPTSEGDFWATTTSDSGLSS 1139

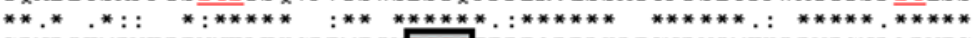

Huma $n$ SRNP I TWPVT PFYNTLT KGPEME I GSGEEREQPEDKDESNPVIWTK I RVPGND APVES 1191

Mouse S-DAMTWQVT PFYSTMTTDPEVE IH GGSG EDSDQPLNKD KSNSVIWNK I GVPEHD APMET 1198

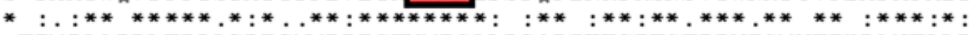

Human -TEMPLAPPLTPDLSRE SWWPPF STVMEGLLPSQRPTTSETGTPRVEGMVTEKPANTLLP 1250 Mouse DAEL PLGPPPTSYMGEE PSWPPF STKMEGSLP---AWSF KNETPRDDGM IAEKSRK I PLP 1255

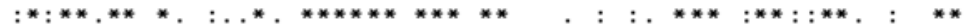

Human LGGDHQPEPSGKTANRNHLKL PNNMNQTKSSEPVL TEEDATS L ITEGF L LNASNY KQLTN 1310

Mouse LAGDHHPATSEKLENHD KLALPNTTNPTQ GF GPVL TEEDASNL I AEGF L LNASDY KHLMK 1315

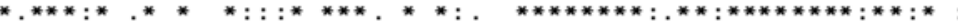

C

(TSR 4) PSLN SL FDL TPAGL LSSNNSAF DP LML I RANSHRQRTVDL DGNDFD IEVR PNQA QP PQ IP IQ PTNSTY DVL DQI SGSGETQTTELNNF TVNMDT I SNTTF DI SGSGSS DI EMSGTT YSTHTL EVTP VNTTNV HVTT PPEPTAPTVQAK ${ }^{138}$ (TSR5)

D

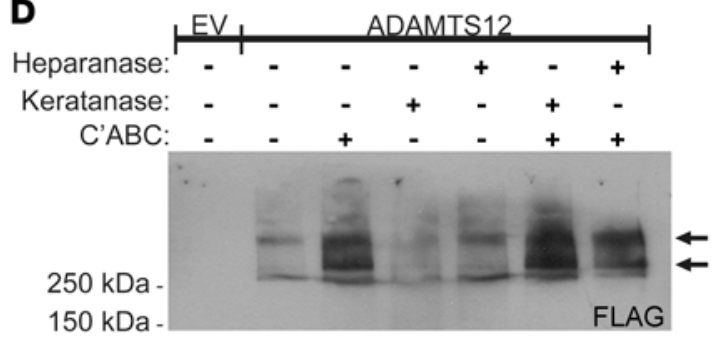

$\mathbf{E}$

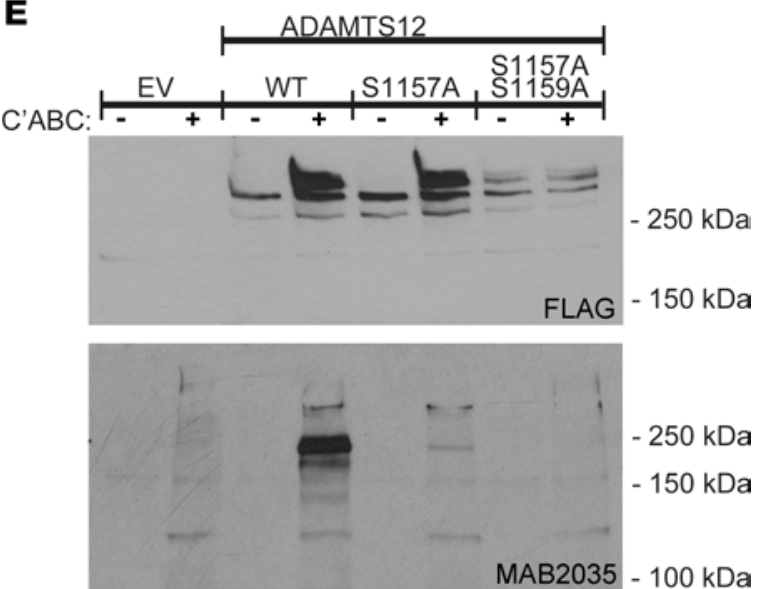

Figure 1. ADAMTS12 is an evolutionarily conserved chondroitin sulfate/dermatan sulfate proteoglycan. (A) Schematic of ADAMTS12 domain structure. TSR, thrombospondin type 1 repeat; CS, chondroitin sulfate. (B) Alignment of human and mouse ADAMTS12 mucin modules showing a high degree of conservation (asterisks show identical residues, residue numbers are shown on the right), potential N-glycosylation sites (bold) and Ser-Cly dipeptide motifs (red) with acidic neighbors, constituting putative glycosaminoglycan attachment sites. (C) Sequence of the corresponding module from the Ciona intestinalis ortholog of ADAMTS7 and ADAMTS12. Sequence motifs for N-glycosylation (bold) and Ser-Gly/Gly-Ser dipeptides (red) are shown. (D) Representative Western blot (anti-FLAG) of conditioned medium from transiently transfected HEK293F cells shows increased ADAMTS12 band intensity (arrows) following digestion with chondroitinase $A B C\left(C^{\prime} A B C\right)$, but not heparanase or keratanase I \& II. The blot is representative of $n=3$. (E) Mutation of Ser ${ }^{1157}$ and Ser ${ }^{1159}$ (boxed residues in panel B), but not Ser ${ }^{1157}$ alone eliminates enhanced signal after C'ABC digestion and reactivity to an anti-chondroitin sulfate stub antibody (MAB2035) strongly suggestive of chondroitin/dermatan sulfate attachment at Ser ${ }^{1157}$ and Ser ${ }^{159}$. EV, empty vector; WT, wild type.

albeit much reduced in the chondroitinase-digested $\operatorname{Ser}^{1157}$ mutant, suggestive of CS/DS-attachment at this site, and none was seen against the $\operatorname{Ser}^{1157+1159} \mathrm{Ala}$ ADAMTS12 mutant, indicating elimination of all CS/DS attachment (Figure 1E; see complete unedited blots in the supplemental material). Thus, like ADAMTS7 (12), ADAMTS12 is a secreted CS/DS PG, with modification occurring at 2 sites, Ser $^{1157}$ and Ser ${ }^{1159}$, indicating their substantial evolutionary conservation as a distinct subfamily.

Adamts 7 and Adamts12 are expressed in hindlimb tendons, meniscal fibrocartilage, and skeletal muscle but not in cartilage. In light of their shared characteristics, the spatial and temporal distribution of Adamts 7 and Adamts12 mRNAs were compared in the developing musculoskeletal system. Forelimbs and hindlimbs 
A

Hindlimb
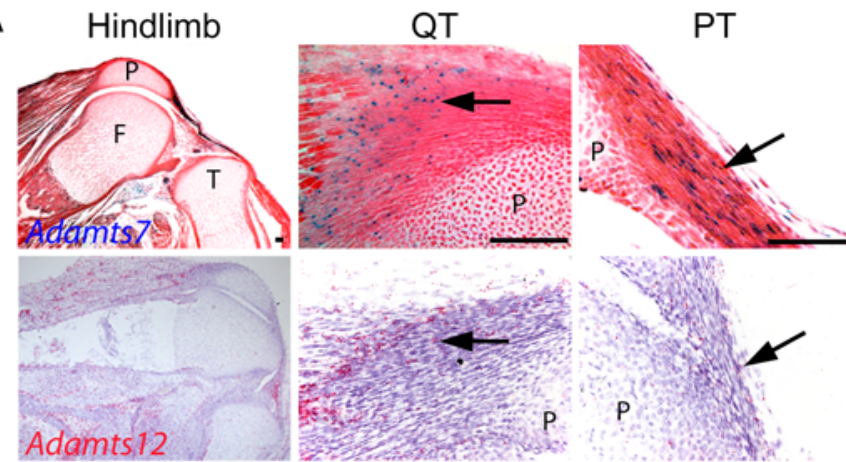

Meniscus
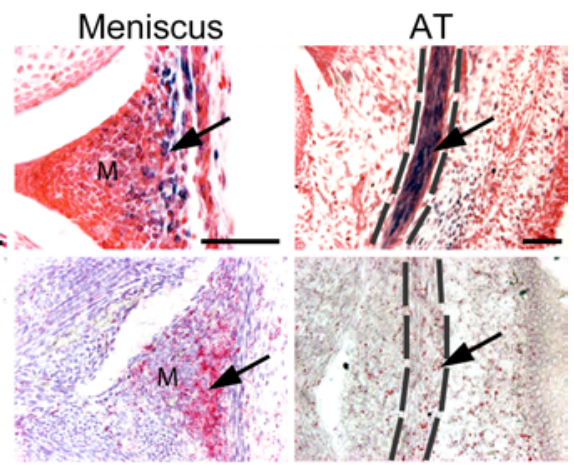

$\mathbf{B}$

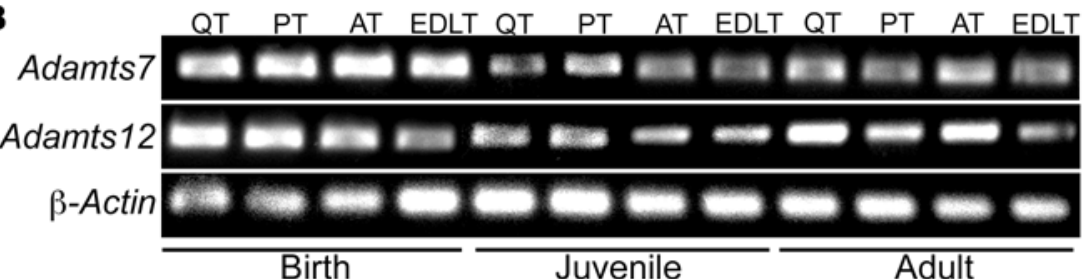

C
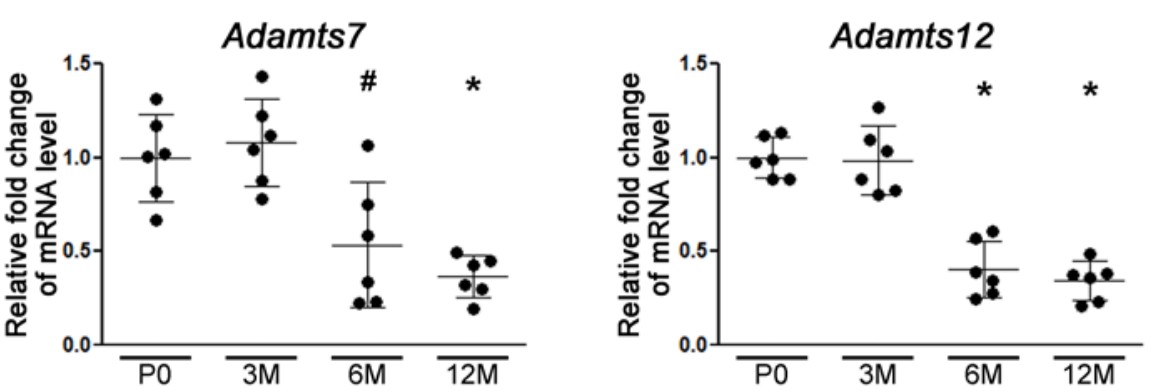

D
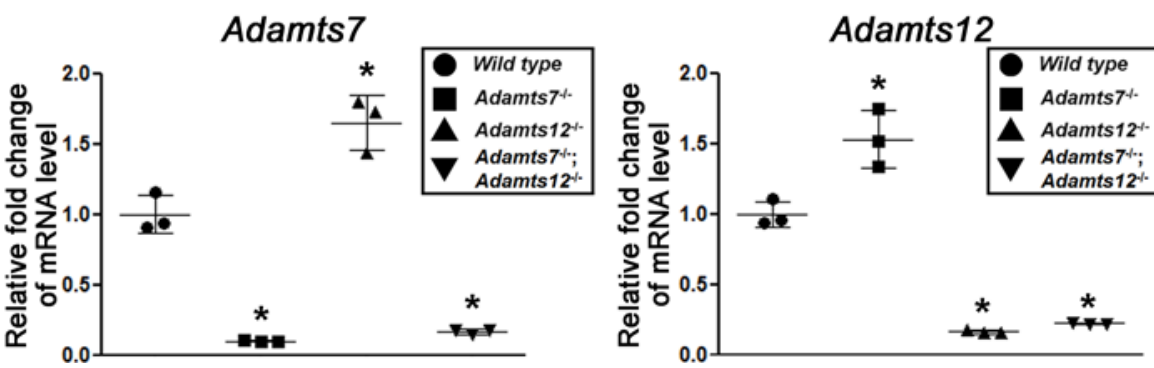

Figure 2. Adamts7 and Adamts12 are expressed in hindlimb tendons and menisci and show mutual compensation. (A) Adamts7 ( $\beta$-gal staining, blue nuclei, eosin counterstain is red) and Adamts12 expression (RNA in situ hybridization [red signal]) are shown in 18.5-day-old embryo hindlimbs. Expression is seen in the quadriceps (QT), patellar (PT), and Achilles tendons (AT) and meniscal fibrocartilage. The data are representative of $n=5$. F, femur; T, tibia; P, patella; M, meniscus. Scale bars: $200 \mu \mathrm{m}$. (B) RT-PCR analysis of newborn, 1-month-old (juvenile), and 4-month-old (adult) tendons to show widespread expression of Adamts7 and Adamts12. EDLT, extensor digitorum longus tendon. The data are representative of $n=3$. (C) qRT-PCR analysis of Adamts7 and Adamts12 mRNA levels in quadriceps tendons showing a decline with age. $n=6$. Error bars represent \pm SEM. (D) qRT-PCR analysis of Adamts7 and Adamts 12 mRNAs in wild-type, Adamts $7^{-/-}$, Adamts $12^{-/-}$, and Adamts $7^{-/-}$Adamts $12^{-/-}$double-knockout mice shows mutual compensation by Adamts7 and Adamts12. $n=3$. Error bars represent \pm SEM. ${ }^{*} P \leq 0.01 ; ~ * P \leq 0.05$ by Student's $t$ test.

from 12.5-, 14.5-, and 18.5-day-old Adamts $7^{+/}$embryos (E18.5) containing all musculoskeletal tissues (cartilage growth plates, articular cartilage, ligaments, tendons, menisci, bone, and skeletal muscle) were stained for $\beta$-galactosidase ( $\beta$-gal) expression as a surrogate for Adamts $7 \mathrm{mRNA}$ in this lac $Z$ insertional allele. Wild-type E18.5 limbs were used for in situ hybridization (ISH) of Adamts12 mRNA. The more extensive analysis of Adamts 7 permitted by the intragenic reporter showed no staining in E12.5 hindlimbs, but emergence in hindlimb tendons at E14.5, and strong staining by E18.5. Both genes were specifically expressed in hindlimb tendons, skeletal muscle, and knee meniscal fibrocartilage but their mRNAs were not detected in articular or growth plate cartilage and bone (Figure 2A and Supplemental Figure 1, A-D; supplemental material available online with this article; https://doi.org/10.1172/jci.insight.92941DS1). 


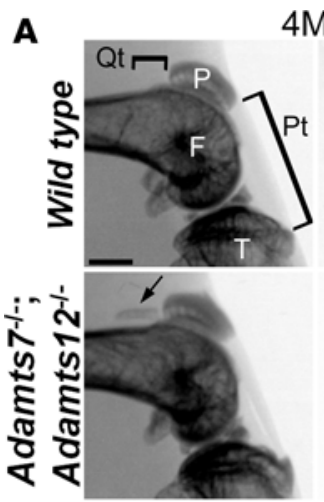

$\mathbf{B}$

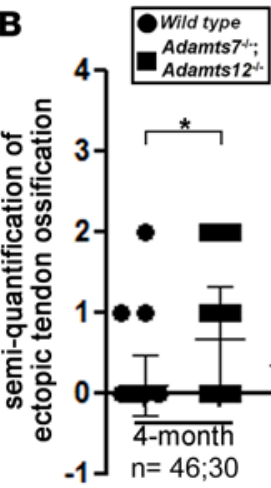

Achilles tendon
$12 \mathrm{M}$
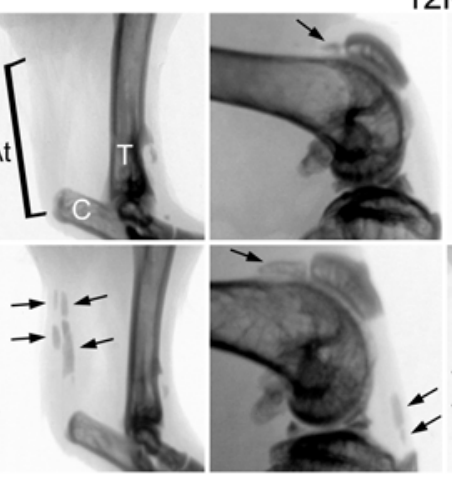
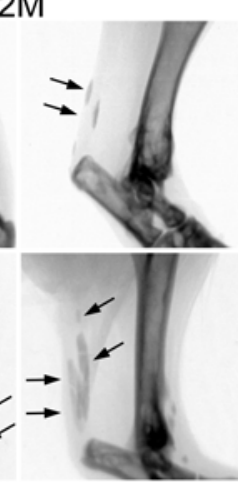
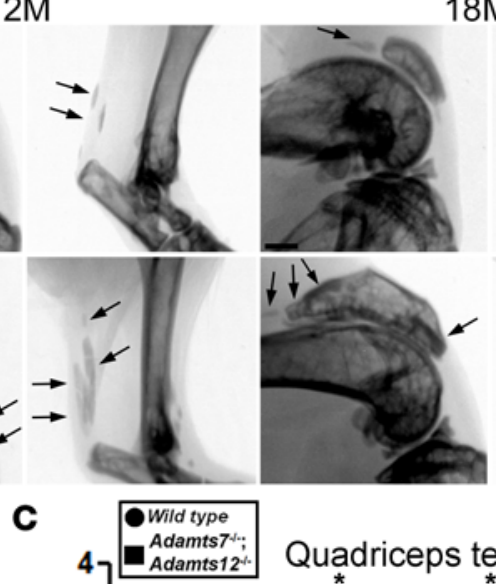

$18 \mathrm{M}$

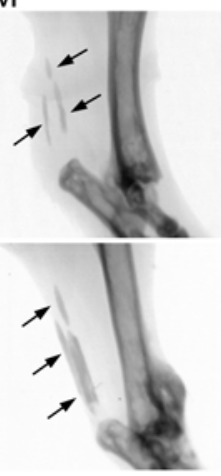

D

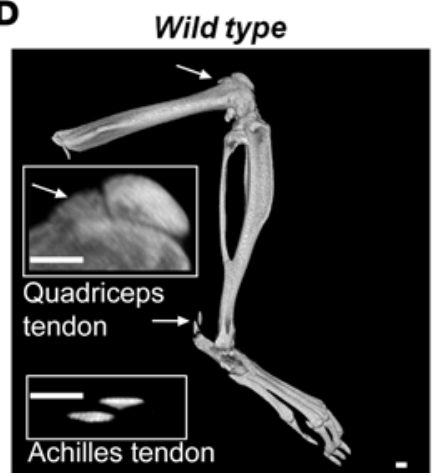

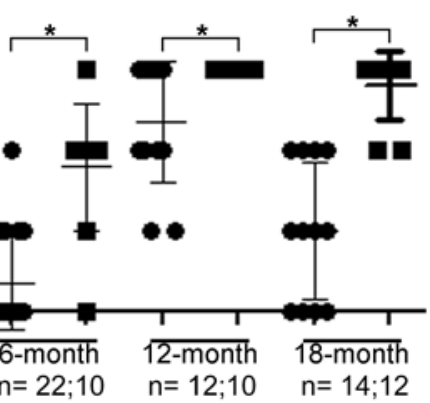

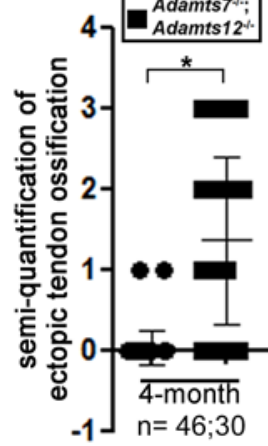

Quadriceps tendon

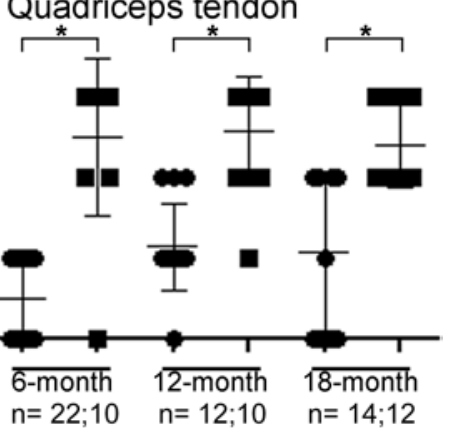

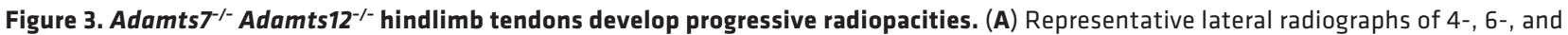
18-month-old (4M, 6M, and 18M) hindlimbs reveal radiopacities (arrows) within the quadriceps tendon (Qt), patellar tendon (Pt), and Achilles tendon (At) of Adamts $7^{-1-}$ Adamts $12^{-/-}$mice that progress with age. Scale bar: $1 \mathrm{~mm}$. P, patella; F, femur; T, tibia; C, calcaneus. (B and C) Semiquantitative scores of heterotopic ossification in Achilles (B) and quadriceps (C) tendons shown in $\mathbf{A}$. (D and $\mathbf{E}$ ) Micro-CT analysis of 18-month-old hindlimbs (scale bars: $1 \mathrm{~mm})(\mathbf{D})$ and quantification of heterotopic ossification (E). $n=4$ Achilles tendon, $n=3$ quadriceps tendons. Error bars represent $\pm \mathrm{SEM}$. ${ }^{*} P \leq$ 0.01 by Student's $t$ test.
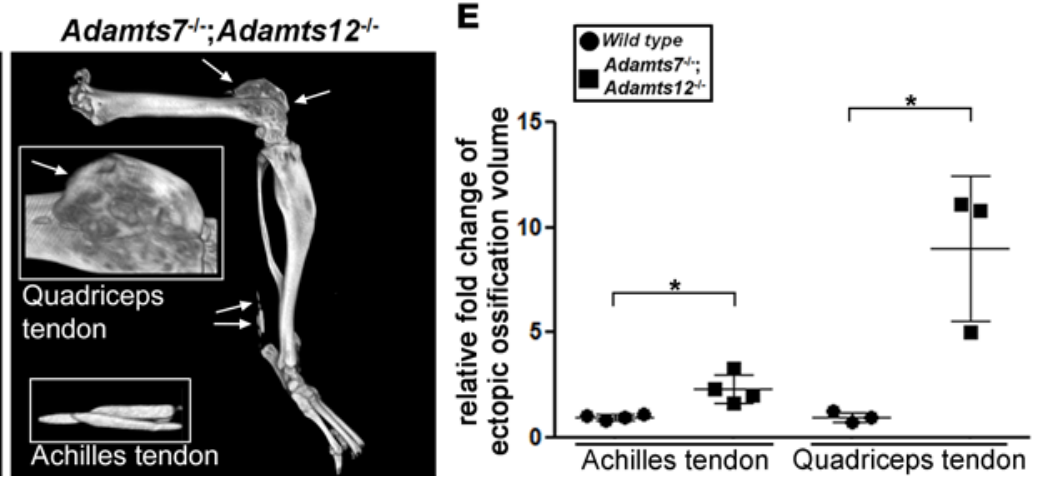

Semiquantitative reverse transcription PCR (RT-PCR) analysis from multiple independent mouse hindlimb tendons at birth, 1 month (juvenile), and 4 months (adult) revealed expression of Adamts 7 and Adamts 12 in the quadriceps, patellar, Achilles, and extensor digitorum longus tendons in juvenile and adult mice (Figure $2 \mathrm{~B}$ ). This was supported by $\beta$-gal staining of adult Adamts $7^{+/-}$limbs (data not shown), demonstrating continuing expression in hindlimb tendons. Adamts7 was expressed in forelimb tendons, but sparsely and with less robust $\beta$-gal staining, whereas Adamts 12 mRNA expression was not detected in forelimb tendons (Supplemental Figure 1B). Longitudinal analysis of Adamts 7 and Adamts12 quadriceps tendon expression by quantitative RT-PCR (qRT-PCR) showed a peak at 3 months of age, with a statistically lower level at 6 months and 12 months (Figure 2C). Thus, hindlimb tendon expression of Adamts 7 and Adamts 12 mRNA is sustained from the embryonic through adult periods, with a gradual decline as mice age. Furthermore, the data showed that these highly homologous metalloproteinases are coexpressed at specific sites during musculoskeletal development, raising the possibility that they could compensate for each other or work 
cooperatively in the same pathways. To ask whether either gene was upregulated in the absence of the other, mRNA from wild-type, Adamts $7^{-1-}$, Adamts $12^{-1-}$, and Adamts $7^{-1-}$ Adamts $12^{-1-}$ quadriceps tendons were analyzed by qRT-PCR at 3 weeks of age. This analysis showed a statistically significant upregulation of Adamts12 in Adamts $7^{-1-}$ tendons and vice versa (Figure 2D), implying compensation in single mutants.

Mice with combined inactivation of Adamts 7 and Adamts12 develop hindlimb tendon, ligament and meniscal heterotopic ossification (HO), but have normal skeletal development. Since overlapping expression and gene compensation could mask the deficiency of either protease alone, compound Adamts $7^{-1}$ Adamts $12^{-1-}$ mice were generated to investigate cooperative functions in musculoskeletal tissues. Adamts $7^{-/}$Adamts $12^{-/-}$mice did not have any externally evident anomalies, were fertile, and did not show enhanced mortality up to 1 year of age. Alizarin red-alcian blue-stained skeletal preparations showed normal skeletal patterning and appropriate endochondral and intramembrane ossification in Adamts $7^{-1-}$ Adamts $12^{-1-}$ mice (Supplemental Figure 2A). Adamts $7^{-1-}$ Adamts12-1- long bones were comparable in length to wild-type long bones (Supplemental Figure 2B), and Adamts $7^{-1-}$ Adamts $12^{-1-}$ mice had similar body weight to that of age-matched wild-type mice (Supplemental Figure 2C). The developing long bones and growth plates were histologically and morphometrically comparable to wild type (Supplemental Figure 2D).

Upon radiographic analysis, radiopacities consistent with $\mathrm{HO}$ were identified within the quadriceps tendon, Achilles tendon, and menisci of 4-month-old Adamts $7^{-1-}$ Adamts $12^{-1-}$ mice (Figure 3A), but in neither wild-type controls nor single-null mutants (Supplemental Figure 3, A-C). The radiopacities progressed with age (Figure 3, A-C) and included patellar tendons in 12-month-old and 18-month-old Adamts $7^{-1-}$ Adamts12 $2^{-/-}$mice, which were not seen in control mice (Figure 3A). Radiopacities were never seen in the forelimb or vertebral tendons (Supplemental Figure 3D). Radiopacities were most extensive in 18-month-old Adamts $7^{-1-}$ Adamts $12^{-/-}$mice, and dense bone within the tendon was observed using micro-CT (Figure 3, D and E). Ectopic mineral was also identified in Adamts $7^{-1-}$ Adamts $12^{-1-}$ hindlimb tendons and ligaments after Alizarin red staining of skeletal preparations at 6 months (Supplemental Figure 4A) and 18 months of age (Figure 4A). Mature lamellar bone was observed in tendon sections, indicating that these mice developed $\mathrm{HO}$ rather than ectopic mineralization (Figure 4B). Adamts $7^{-1-}$ Adamts $12^{-/}$-mice did not develop osteoarthritis, as indicated by an intact superficial articular cartilage zone and underlying articular cartilage as late as 18 months of age (Supplemental Figure 4B), and subchondral bone thickness and volume were unaltered at 18 months of age, also suggestive of a lack of joint degeneration (Supplemental Figure 4, C and D).

While tendon markers such as scleraxis $(S c x)$, mohawk $(M k x)$, tenomodulin (Tnmd), collagen 1 (Col1a1), and collagen 3 (Col3a1) were unchanged, markers of cartilage, namely aggrecan (Acan), hypertrophic chondrocyte marker collagen X (Col10a1), and bone markers runt-related transcription factor 2 (Runx2) and osterix ( $S p 7$ ) were all expressed at higher levels in 3-month-old Adamts $7^{-1-}$ Adamts12-/- quadriceps tendons (Figure $4 \mathrm{C}$ ). Stronger immunolocalization of aggrecan and collagen $\mathrm{X}$, markers of the chondrocyte and hypertrophic chondrocyte phenotype, respectively, was observed in quadriceps tendon (Figure 4D). Quadriceps tendon contains some fibrocartilage, explaining the low level of aggrecan staining observed in wild-type quadriceps, but the local expression of collagen $\mathrm{X}$ in Adamts $7^{-1-}$ Adamts12-1quadriceps tendons, taken together with qRT-PCR data (Figure 4C), strongly supports local progression toward $\mathrm{HO}$ by endochondral ossification (Figure 4D).

Abnormal tendon collagen fibrils in Adamts7 $7^{-1}$ Adamts12-- mice. Codistribution of Adamts7 and Adamts12 mRNAs within hindlimb tendons, whose ECM comprises tightly packed collagen fibrils oriented uniaxially along the tendon long axis, plus the importance of PGs in tendon collagen fibrillogenesis (22-24), prompted investigation of collagen ultrastructure in Adamts $7^{-1-}$ Adamts $12^{-1-}$ tendons. Collagen fibril structure and diameter distribution were analyzed in Adamts $7^{-1-}$ Adamts $12^{-/-}$Achilles tendons compared with wild-type Achilles tendons using transmission electron microscopy (TEM). In contrast to collagen fibrils of 1-month-old wild-type Achilles tendon, which had a smooth, circular cross-sectional profile and a bellshaped distribution of fibril diameter, Adamts $7^{-1-}$ Adamts $12^{-/-}$tendons had a higher frequency of both large diameter fibrils and fibrils with abnormal outlines (Figure $5 \mathrm{~A}$ ). Whereas a change in fibril area or density was not observed (Figure 5B), quantitative analysis of the fibril diameters revealed statistically significant changes in their size range, mean diameter, and distribution profile (Figure 5C). On average, Adamts $7^{-1-}$ Adamts $12^{-1-}$ tendon fibrils had larger diameters than control fibrils and there was a reduction in the number of small fibrils. These findings indicated dysregulation of collagen fibrillogenesis in the absence of Adamts 7 and Adamts12 leading to altered lateral fibril growth. 

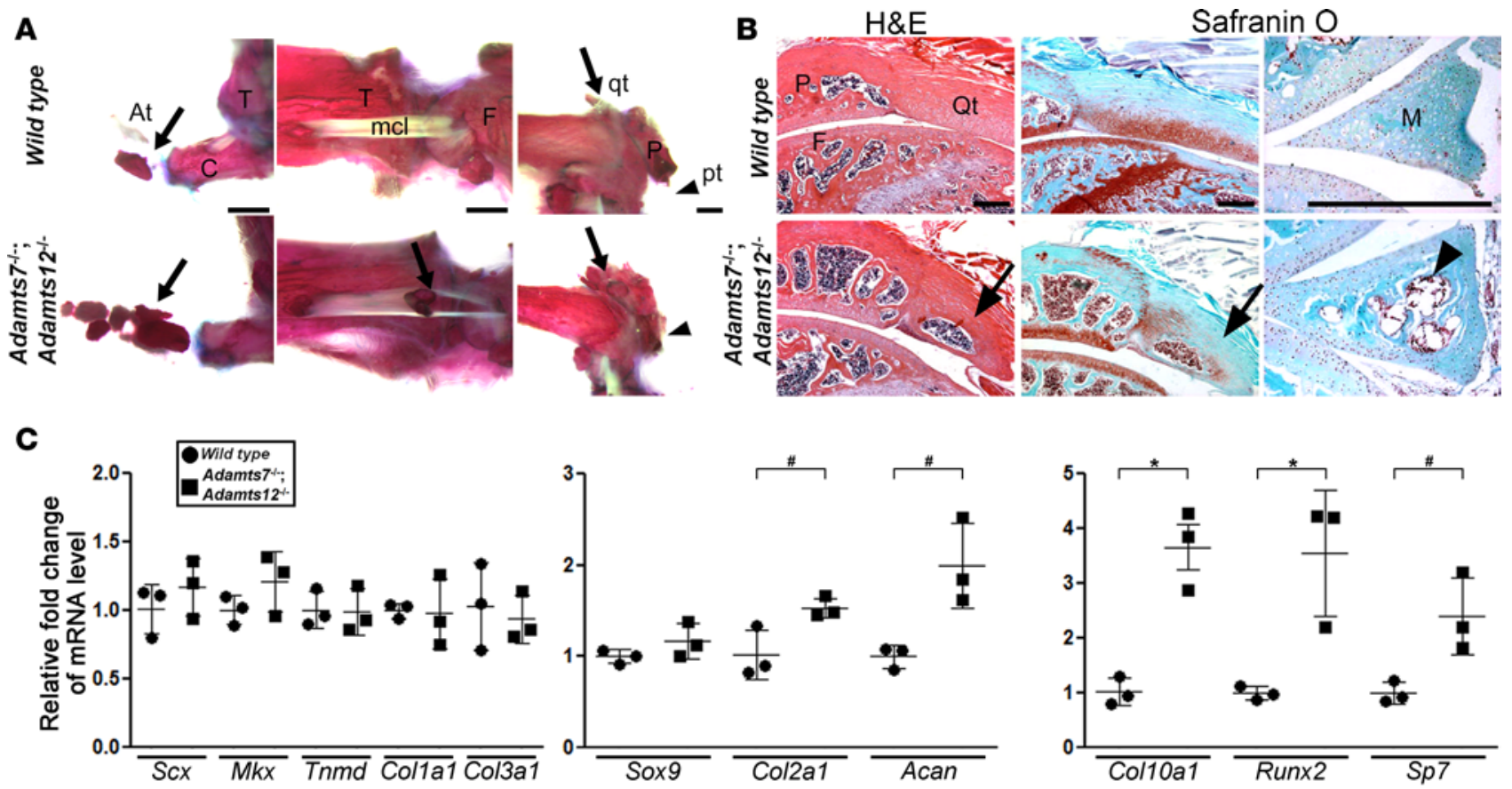

D

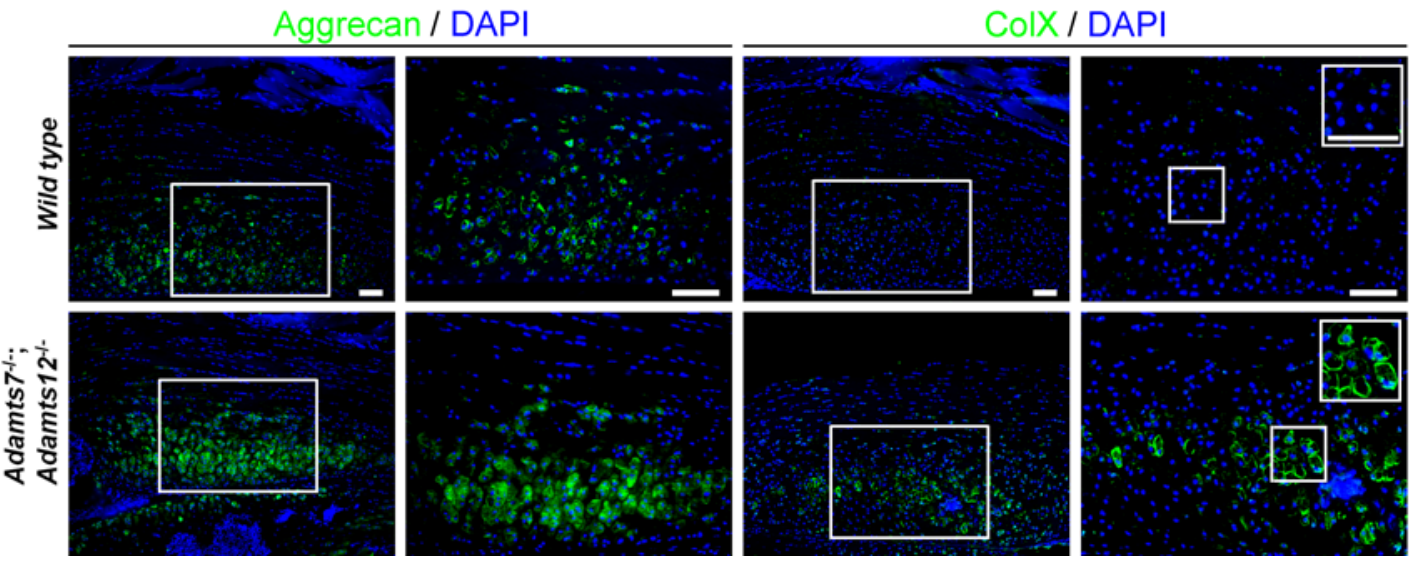

Figure 4. Adamts7 $7^{-/-}$Adamts12-/- hindlimbs develop heterotopic tendon and ligament ossification. (A) Alizarin red-Alcian blue-stained skeleton preparations of 18-month-old wild-type and Adamts $7^{-1-}$ Adamts $12^{-1-}$ hindlimbs reveal heterotopic ossification (arrows and arrowheads) in Achilles tendon (At), medial collateral ligament (mcl), patellar tendon (pt), and quadriceps tendon (qt). Data are representative of $n=8$. F, femur; C, calcaneus; T, tibia. (B) $\mathrm{H} \& \mathrm{E}$-stained and safranin $\mathrm{O}$-stained 4-month-old Adamts $7^{-1-}$ Adamts $12^{-1-}$ hindlimbs shows ectopic ossification in the quadriceps tendon (Qt, arrows) and an ossified meniscus (M). F, femur; P, patella. The images are representative of $n=5$. (C) qRT-PCR analysis of 3-month-old wild-type and Adamts7-1Adamts12-/ quadriceps tendons. $n=3$. Error bars represent \pm SEM. ${ }^{*} P \leq 0.01,{ }^{*} P \leq 0.05$ by Student's $t$ test. (D) Increased staining intensity (green) of aggrecan and collagen X (ColX) in 3-month-old Adamts $7^{-1-}$ Adamts12 $2^{--}$quadriceps tendons. Sections were counterstained with DAPI (blue). Data are representative of $n=4$. Boxed images are shown enlarged on the right. Scale bars: $1 \mathrm{~mm}(\mathbf{A})$ and $50 \mu \mathrm{m}$ (B and D).

Reduced small leucine-rich PG content of Adamts $7^{-1-}$ Adamts12-1- tendons may underlie abnormal collagen fibrillogenesis. Previous work indicated that small leucine-rich PGs (SLRPs) were required for proper tendon collagen fibril assembly and function and the fibril data obtained here are consistent with SLRP involvement $(27,28)$. In particular, tendon collagen fibril anomalies in combination with hindlimb $\mathrm{HO}$ were previously seen in $B g n$ ${ }^{10} \mathrm{Fmod}^{-1-}$ mice with combined loss of the SLRPs biglycan and fibromodulin $(22,23,29)$. Reduced protein levels of biglycan, fibromodulin, and decorin were observed in 3-month-old Adamts $7^{-1}$ Adamts $12^{-/-}$quadriceps and patellar tendons compared with wild-type tendons using immunohistochemistry (Figure 6A and Supplemental Figure 5, A-C) and Western blot analysis (Figure 6B; see complete unedited blots in the supplemental material). The reduced quadriceps tendon content of biglycan, fibromodulin, and decorin appears to have a posttranscriptional basis, since qRT-PCR showed no statistically significant difference between the respective 
A

Wild type

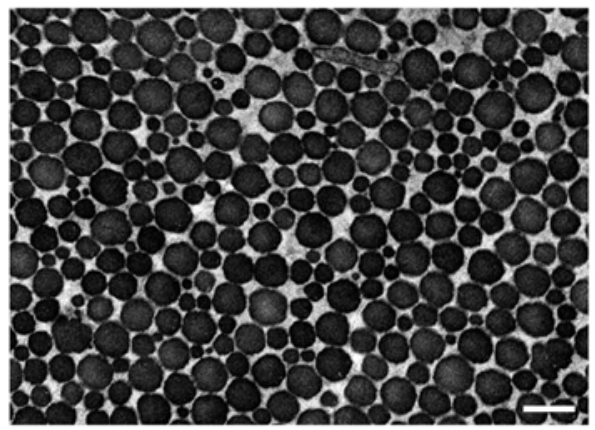

B

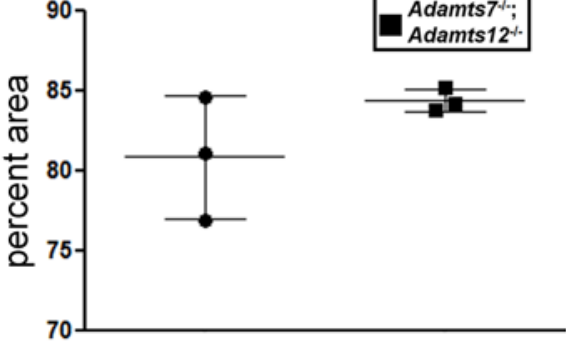

Fibril Area

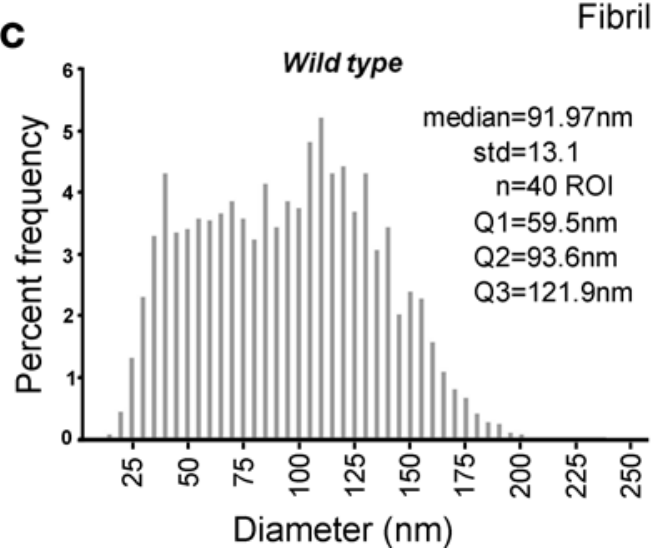

Adamts7"-;Adamts12\%

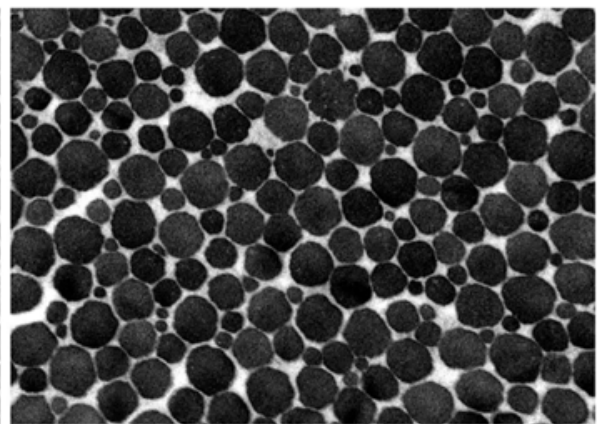

Fibril Density

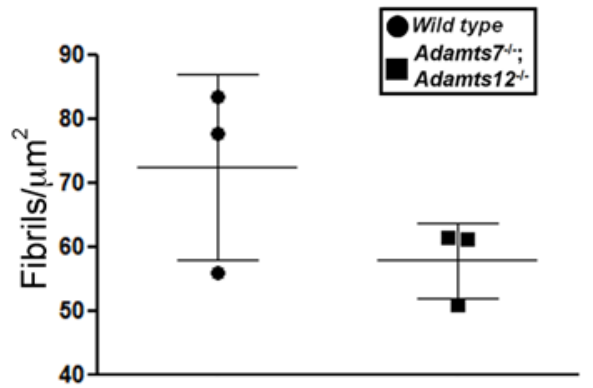

Fibril Diameter

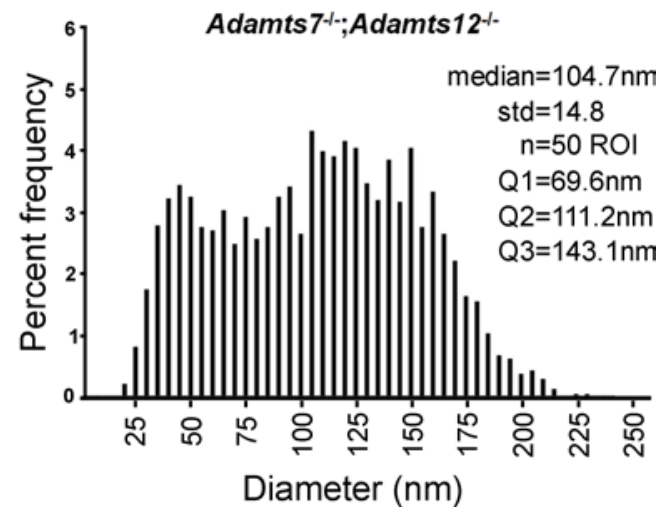

Figure 5. Transmission electron micrographs of Achilles tendon reveal thicker collagen fibrils in Adamts7 ${ }^{-/-}$Adamts12 ${ }^{-/-}$mice. (A) Representative transverse sections of 21-day-old Adamts7/- Adamts12-/- Achilles tendons reveals larger collagen fibrils than in wild-type tendons. The images are representative of $n=3$. Scale bar: $200 \mathrm{~nm}$. (B) No change in percentage fibril area, but a reduced percentage of tendon fibril density was evident in Adamts7 $7^{-/-}$Adamts $12^{-/-}$tendons. Error bars represent \pm SEM. Significance was determined by Student's $t$ test. (C) Histograms representing fibril diameter distribution. Adamts7 $7^{-/-}$Adamts $12^{-/-}$tendons have a broader distribution of fibril diameters, with an increase in large-diameter fibrils. Std, standard deviation; ROI, region of interest; Q, quartile.

mRNAs in mutant and wild-type tendons (Figure 6C). COMP was previously identified as an ADAMTS7 and ADAMTS12 substrate $(17,18)$, but immunostaining did not reveal consistent alteration in COMP staining (Supplemental Figure 5D). Because reduced tendon biglycan and decorin were previously associated with increased BMP signaling, 3-month-old (i.e., prior to overt $\mathrm{HO}$ ) wild-type and mutant quadriceps tendons were immunostained using antibodies against p-SMAD1/5/9. More extensive staining was seen in Adamts $^{-1-}$ Adamts $12^{-1-}$ quadriceps tendons (Figure 6D), suggestive of enhanced BMP signaling.

Suppression of chondrocyte differentiation of uncommitted progenitors by ADAMTS7 and ADAMTS12 could underlie $H O$. Chondrogenesis is a multistep process initiated by mesenchymal cell condensation in uncommitted precursors. The emergence of $\mathrm{HO}$ in Adamts $7^{-1-}$ Adamts $12^{-1-}$ hindlimb tendons via an endochondral ossification program suggested a physiological, local role for ADAMTS7 and ADAMTS12 in suppressing chondrogenesis. To determine whether Adamts 7 and Adamts12 could inhibit chondrogenesis, the impact of 
A
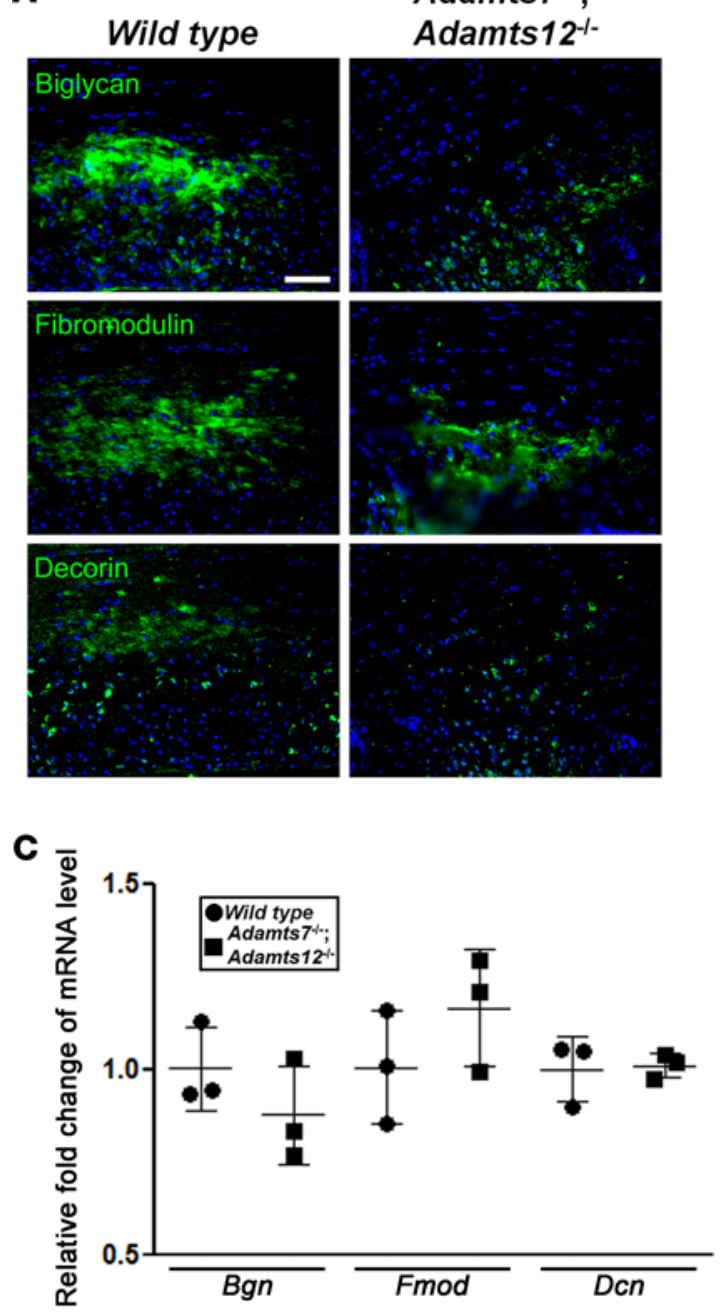

B

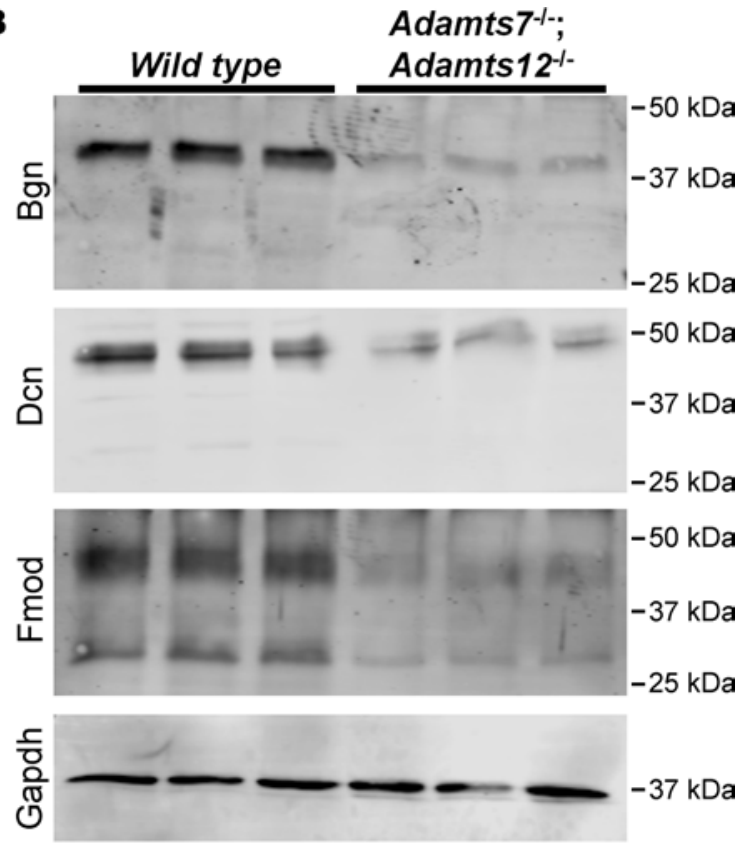

D

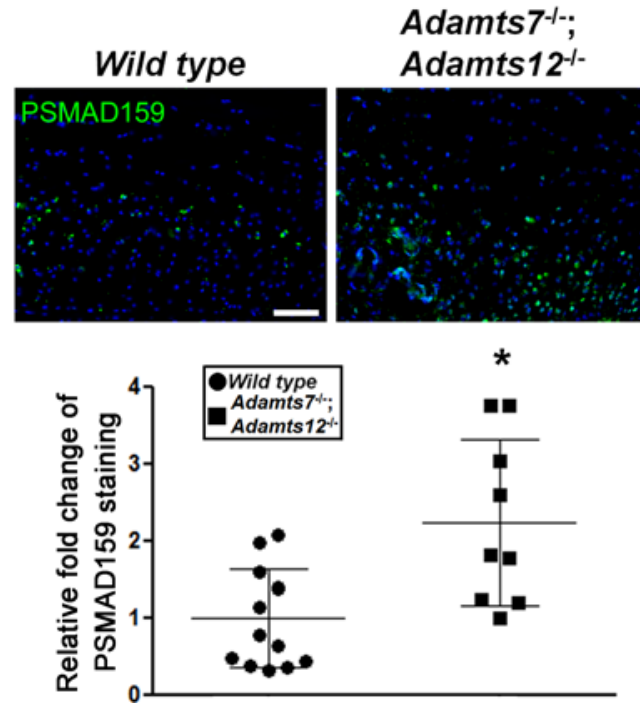

Figure 6. Reduction of small leucine-rich proteoglycans in Adamts7-/- Adamts12-/- quadriceps tendons. (A) Reduced staining intensity (green) of biglycan, fibromodulin, and decorin is seen in 3-month-old Adamts $7^{-1-}$ Adamts $12^{-/-}$quadriceps tendon. Sections were counterstained with DAPI (blue). Data are representative of $n=4$. (B) Western blots show reduced biglycan (Bgn), fibromodulin (Fmod), and decorin (Dcn) content in 3-month-old Adamts7-/Adamts $12^{-/-}$quadriceps tendons $(n=3)$ compared with wild-type tendons $(n=3)$. (C) qRT-PCR analysis of 3-month-old wild-type and Adamts $7^{-1-}$ Adamts12-/- quadriceps tendons. $n=3$. (D) Increased staining of $p-5 m a d 1 / 5 / 9$ (green) in 3-month-old Adamts $7^{-1-}$ Adamts $12^{-/-}$quadriceps tendons. Sections were counterstained with DAPI (blue) and quantified below. $n=12$ wild type, $n=9$ Adamts $7^{-1-}$ Adamts $12^{-/}$. Scale bars: $50 \mu \mathrm{m}$. Error bars represent \pm SEM. ${ }^{*} P \leq 0.01$ by Student's $t$ test.

their overexpression was investigated in $\mathrm{C} 3 \mathrm{H} 10 \mathrm{~T} 1 / 2$ cells, a pluripotent mouse embryo cell line (30). This cell line can be directed to the chondrocyte lineage by culture under high-density conditions, such as micromass culture or pellet culture in chondrogenic medium (Figure 7A) $(31,32)$. Adamts 7 and Adamts 12 mRNAs are expressed by $\mathrm{C} 3 \mathrm{H} 10 \mathrm{~T} 1 / 2$ cells at very low levels (Figure 7B); their expression was elevated by transfection with Adamts 7 or ADAMTS12 constructs and maintained in chondrogenic medium (Figure 7, A and B). qRTPCR analysis of the pellet and micromass cultures showed that expression of the cartilage marker Col2al was reduced from 2-7 days of culture in ADAMTS7- or ADAMTS12-overexpressing cells, implying delayed chondrogenesis (Figure 7C and data not shown). Subsequently, chondrocyte differentiation (hypertrophy), as determined by Col10a1 expression, was reduced at days 7-10 in overexpressing cells compared with the 
A

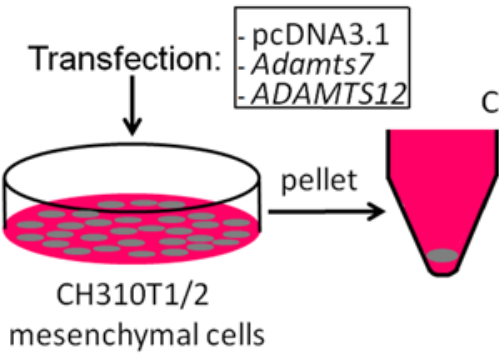

C

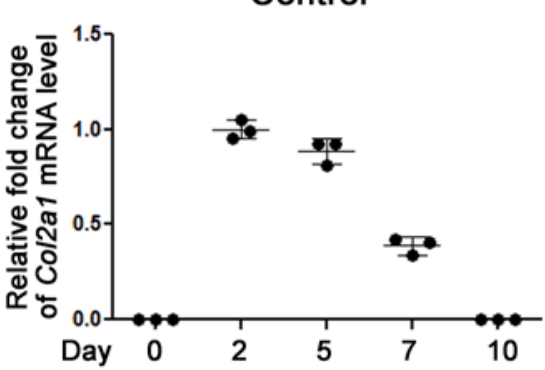

D

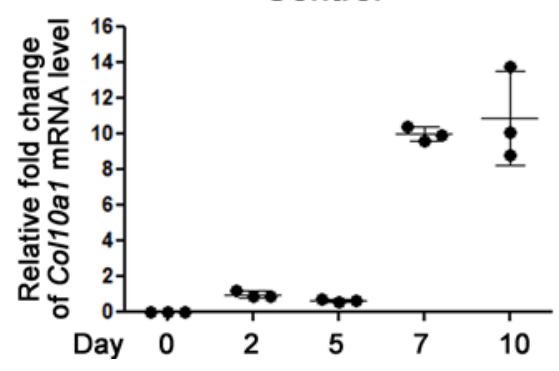

B

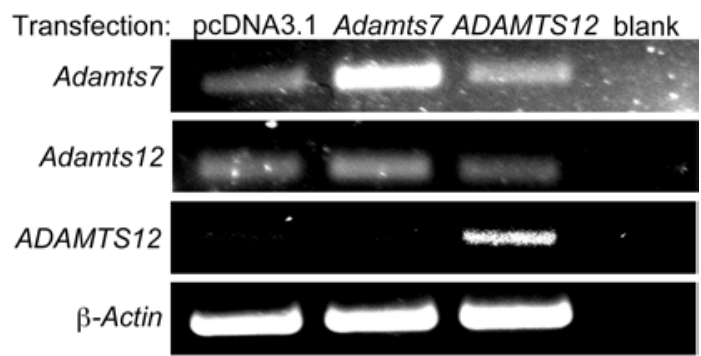

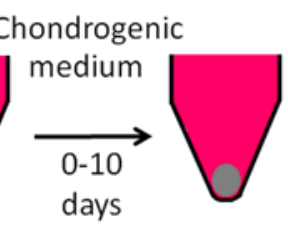

Adamts7

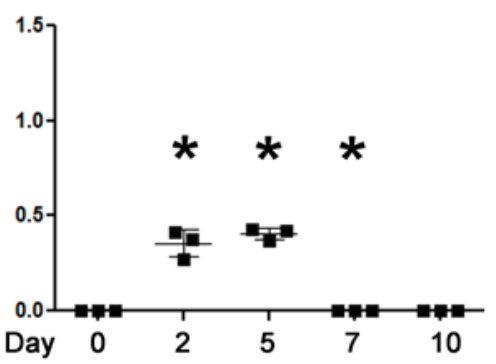

Adamts7

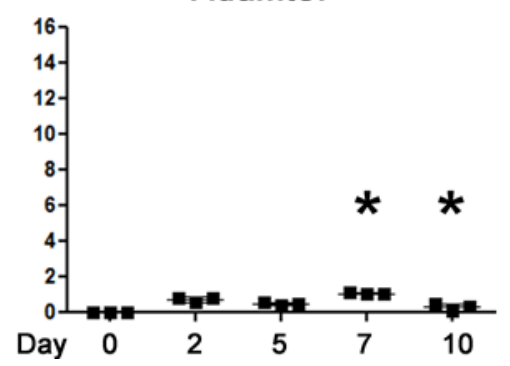

ADAMTS12

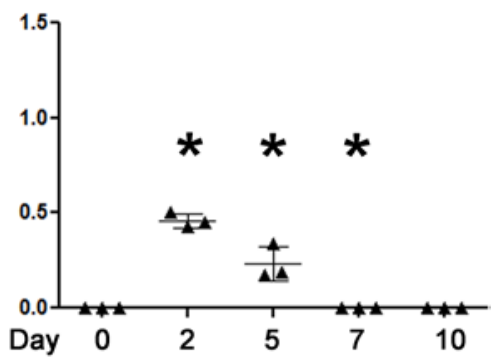

ADAMTS12

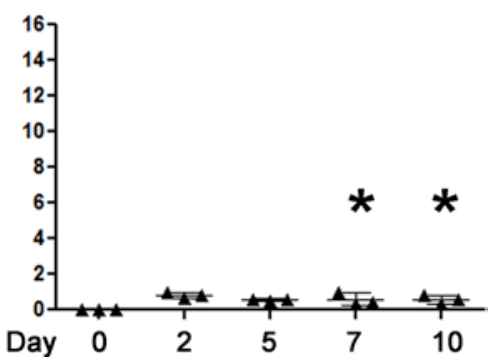

Figure 7. ADAMTS7 and ADAMTS12 overexpression inhibits chondrocyte differentiation in uncommitted C3H10T1/2 cells. (A) Schematic of chondrogenic culture conditions used for 10T1/2 cells. (B) qRT-PCR showing baseline Adamts7 and Adamts 12 expression in C3H10T1/2 cells and overexpression following transfection with the respective plasmids. $n=3$. (C and D) Col2a1 and Col10a1 expression was reduced upon overexpression of Adamts7 or ADAMTS12. $n=$ 3. Error bars represent $\pm \mathrm{SEM}$. ${ }^{*} P \leq 0.01$ by Student's $t$ test.

controls (Figure 7D). There was no change in Bgn, Fmod, or Dcn expression as compared with ADAMTS7- or ADAMTS12-overexpressing cells (Supplemental Figure 6, A-C). Therefore, forced expression of Adamts7 and ADAMTS12 can suppress differentiation of uncommitted mesenchymal cells to the chondrocyte lineage.

ADAMTS7 and ADAMTS12 expression is a hallmark of healthy human tendons. To ask whether ADAMTS7 and ADAMTS12 played a role in tendon phenotype maintenance and overall tendon health in humans, ADAMTS7 and ADAMTS12 mRNA levels were measured in human biceps long head tendons resected during shoulder reconstructive surgery. The tendons were grouped into 2 categories, normal and degenerative, based on their gross appearance and on the basis of normal histology versus a histologic appearance of disorganized structure, staining for sulfated PGs or HO, respectively (Figure 8A). A subpopulation of such degenerative tendons, but not visually normal tendons, showed overt chondrogenesis, containing cells with a chondrocytic morphology and strong GAG staining, or showed endochondral ossification (Figure 8B, arrows). Reduced ADAMTS7 and ADAMTS12 immunoreactivity was evident in degenerative tendons (Figure 8C). ADAMTS7 and ADAMTS12 mRNAs were consistently present in normal human biceps tendons. However, ADAMTS7 mRNA levels showed a statistically significant reduction, and ADAMTS12 mRNA was reduced, albeit not significantly, in degenerative tendons (Figure 8D). Interestingly, as with the Adamts $7^{--}$Adamts $12^{--}$and wild-type tendons, there was not a detectable difference in $B G N$, FMOD, or DCN expression between the 2 tendon groups (Supplemental Figure 7). Therefore, ADAMTS7 and ADAMTS12 could be biomarkers for the healthy tendon state, and their loss could be causally related to some aspects of tendon degeneration, such as ectopic chondrogenesis and HO. 
A

Normal
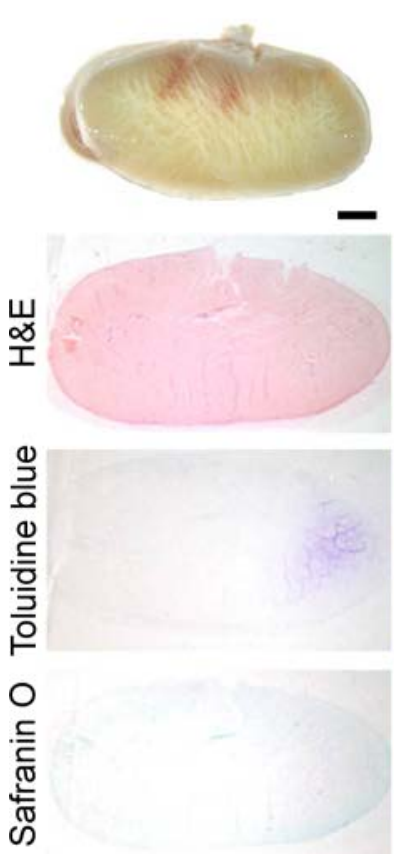

C

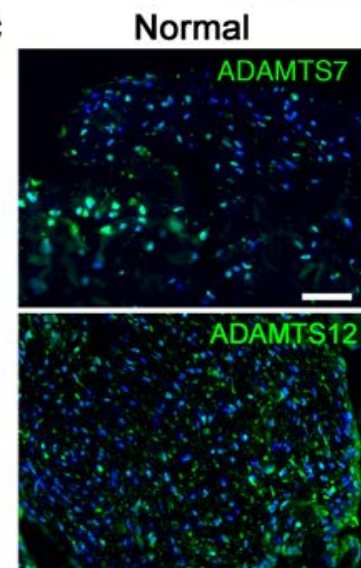

Degenerative
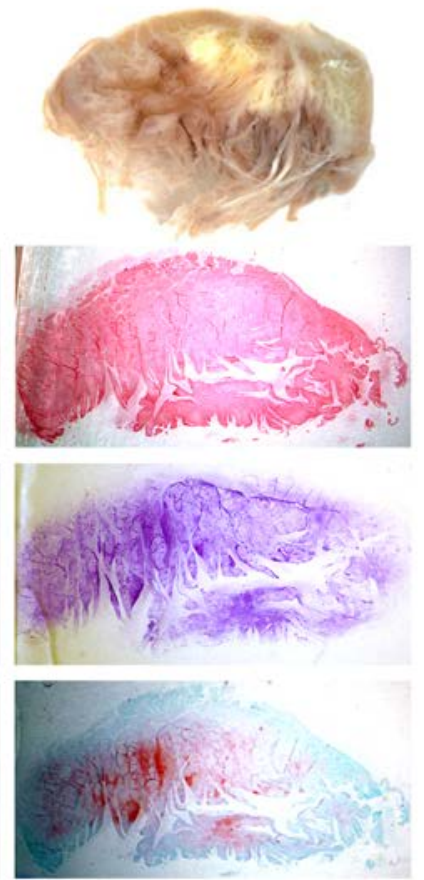

Degenerative

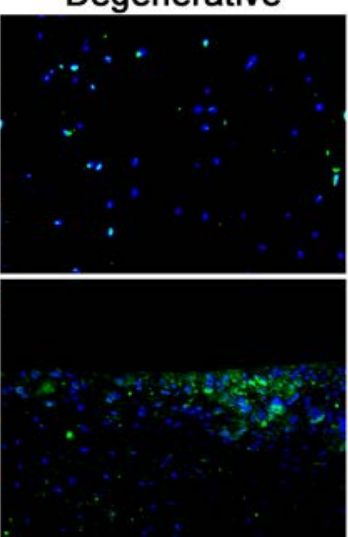

B

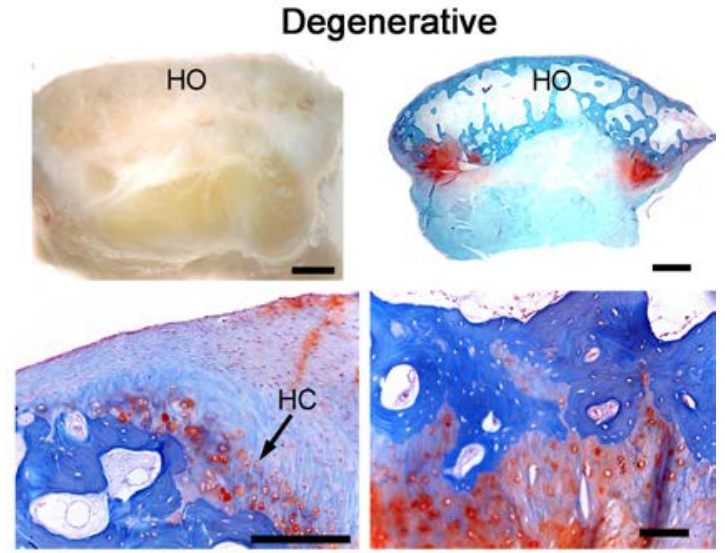

D

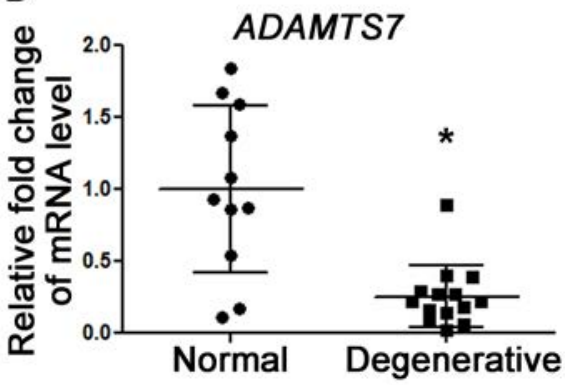

ADAMTS12

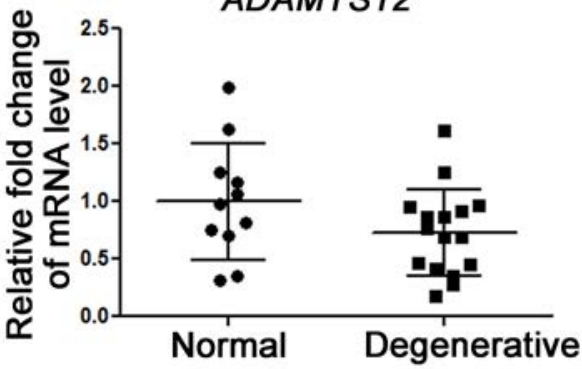

Figure 8. ADAMTS7 and ADAMTS12 mRNA and protein are reduced in degenerative adult human biceps tendons. (A) Gross appearance of cross sections of histologically normal and degenerative adult human biceps tendon (top) and overview of staining with H\&E and proteoglycan stains toluidine blue and safranin 0 as indicated. Images are representative of $n=10$ normal and $n=15$ degenerative. (B) An example of a degenerative tendon stained with safranin $\mathrm{O}$ showing heterotopic ossification ( $\mathrm{HO}$ ) and the appearance of hypertrophic chondrocytes (HCs). The images are representative of $n=3$ tendons with $\mathrm{HO}$. (C) Representative immunostaining of normal and degenerative tendon sections with anti-ADAMTS7 (top panels, green) and anti-ADAMTS12 (lower panels, green). Images are representative of $n=3$. (D) qRT-PCR reveals ADAMTS7 and ADAMTS12 mRNA expression in histologically normal human biceps tendons and significant reduction of ADAMTS7 mRNA in histologically degenerative tendons. 10 normal and 15 tendons with degenerative change were used for the analysis. Scale bars: $1 \mathrm{~mm}$ (A and upper panels in B), $200 \mu \mathrm{m}$ (lower panels in B), and $50 \mu \mathrm{m}$ (C). Error bars represent \pm SEM. ${ }^{*} P \leq 0.01$ by Student's $t$ test.

\section{Discussion}

The present work, together with a prior report (12), establishes that ADAMTS7 and ADAMTS12 comprise a distinct subfamily of ADAMTS proteases that are also CS/DS PGs. DS differs from CS in the presence of CS disaccharides containing not only D-glucuronate, which is characteristic of CS but its 5-epimer, L-iduronate (33). Chondroitinase ABC digestion cannot distinguish between the two, and chondroitinase ACII, which can, is presently commercially unavailable. CS/DS PGs have specific structural and instructive roles in many tissues. For example, aggrecan, which has numerous CS chains, provides cartilage with resistance to compression (34). CS chains can mediate binding to morphogens and growth factors such as sonic hedgehog, platelet-derived growth factor, and TGF- $\beta(35,36)$.

The unusually close molecular relationship and observed compensatory upregulation of Adamts 7 or 
Adamts12 in the absence of the other led us to generate and analyze mice lacking both ADAMTS7 and ADAMTS12 for the first time time to the best of our knowledge. Previously, mouse mutants with combined deficiency of ADAMTS5, ADAMTS9, or ADAMTS20, proteases that are related by their ability to cleave the CS PG versican and are expressed coordinately in interdigital mesenchyme, manifested soft tissue syndactyly with a higher penetrance than any of the single mutant alleles (37). In addition, Adamts $20^{\text {bt }}$ ${ }^{b t}$ Adamts ${ }^{+/-}$mice, which are deficient in highly homologous ADAMTS proteases, have fully penetrant cleft palate, whereas Adamts $20^{b t / b t}$ mice have a low penetrance and Adamts $9^{+/-}$mice never develop cleft palate (37, 38). The present study extends the concept that closely related ADAMTS proteases work cooperatively, since the combined mutants, but neither single mutant, developed HO.

ADAMTS7 and ADAMTS12 expression was previously reported in bone, cartilage, synovium, ligament, and tendon using RT-PCR $(17,18)$. However, the precise spatial and temporal expression of their mRNAs and roles in skeletal development and adult osteoarticular homeostasis in vivo were previously undefined. The present report herein establishes that neither skeletal patterning nor establishment of specific musculoskeletal lineages is perturbed in Adamts $7^{-/}$Adamts $12^{--}$embryos, since they have the normal number and appropriate organization of limb segments and rays, and properly formed musculoskeletal tissues at each developmental stage. The findings are consistent with the observed absence of significant Adamts 7 and Adamts 12 mRNA expression in cartilage during the embryonic and juvenile periods using RNA ISH and intragenic reporters, which have high sensitivity and specificity in pinpointing the precise source of secreted molecules. The double mutants used here provided a definitive in vivo model for analysis of both related proteins in $\mathrm{HO}$ that was previously unavailable and unequivocally contradict proposed roles for these proteases in endochondral ossification $(39,40)$.

After skeletal maturity, specifically, by 4 months of age, Adamts $7^{-1-}$ Adamts $12^{-1-}$ mice consistently developed HO in hindlimb tendons and ligaments, typically in fibrocartilage within these tissues. Both the peripheral third of the meniscus and the insertion sites of large tendons and ligaments such as the Achilles tendon and quadriceps tendon, as well as the medial collateral ligament contain fibrocartilage, which provides a structural and mechanical transition to bone. Fibrocartilage has an ambiguous histologic and molecular identity, containing both collagen I for tensile strength and aggrecan and other cartilage molecules for compressive strength. Although the cells embedded within fibrocartilage appear chondrocytic, they do not exclusively express chondrocyte or fibroblast proteins. We suggest that fibrocartilage represents a "commitment tightrope," which requires a balance of prochondrogenic and profibrogenic factors within these cells and that ADAMTS7 and ADAMTS12 are profibrogenic factors that are coexpressed locally to maintain the fibrocartilaginous or tendinous state. In the absence of ADAMTS7 and ADAMTS12 in tendons, the transition to cartilage and bone via endochondral ossification, which appears to be part of a normal aging process in C57BL/6 tendons, is expedited. In support, overexpression of Adamts7 and ADAMTS12 in C3H10T1/2 cells grown in pellet cultures suppressed chondrogenesis, confirming similar experiments performed in $\mathrm{C} 3 \mathrm{H} 10 \mathrm{~T} 1 / 2$ micromass cultures $(39,40)$. Together, these findings support an antichondrogenic role in tendons and fibrocartilage.

Interestingly, $\mathrm{Bgn}^{-10} \mathrm{Fmod}^{-1-}$ mice develop hindlimb $\mathrm{HO}$ that is remarkably similar in its spatial distribution to that observed in Adamts $7^{-/-}$Adamts $12^{-/-}$mice, but appears earlier, is more extensive and accompanied by altered gait and premature joint degeneration, which were not evident in the present work $(29,41,42)$. Both SLRPs were reduced in Adamts $7^{-1-}$ Adamts $12^{-/-}$tendons, through mechanisms that are not presently understood, but appear to be nontranscriptional, suggesting that reduced biglycan and fibromodulin could underlie the HO. $\mathrm{Bgn}^{-10} \mathrm{Fmod}^{-1-}$ mice have more tendon progenitor cells and reduction of biglycan and fibromodulin upregulated BMP signaling (43). BMP signaling inhibits tendon formation during development (44), whereas increased BMP signaling is associated with HO (45). Adamts $7^{-/-}$Adamts $12^{-/-}$mice had reduced, rather than absent biglycan and fibromodulin, which could explain why the observed anomalies were not as severe as in $\mathrm{Bgn}^{-10} \mathrm{Fmod}^{-1-}$ mice, and like them, showed upregulated BMP signaling at sites of HO. That PG-deficient mouse models, including $B g n^{-10}$ Fmod ${ }^{-1-}$ and Adamts $7^{-1-}$ Adamts $12^{-/-}$mice develop $\mathrm{HO}$ in tendons supports the concept that these PG components of the ECM have important roles in controlling tendon stem/progenitor cell fate and function (41). The collagen fibril phenotype of Adamts $7^{-/-}$Adamts $12^{-/-}$mice was not, however, identical to those of $\mathrm{Bgn}^{-10} \mathrm{Fmod}^{-1-}$ mice (29), possibly explained by reduction also of decorin, another fibrillogenesis regulator (23). The distinct collagen fibril anomaly of Adamts $7^{-/}$Adamts $12^{-/-}$tendons likely reflects a complex, combined deficiency of SLRPs. As PGs, ADAMTS7 and ADAMTS12 may also directly bind maturing collagen fibrils and regulate their lateral growth. 
Inactivation of the transcription factor Mohawk also leads to tendon $\mathrm{HO}$, but this occurred via failed tenogenesis (46). Neither Adamts 7 nor Adamts12 is a known downstream target of Mohawk, and tenogenesis was unaffected in Adamts $7^{-1}$ Adamts $12^{-/-}$mice, which lacked developmental anomalies. Interestingly, sesamoid bones form during development by endochondral ossification within predetermined regions of tendons (47). Clearly, the intrinsic mechanism that prevents HO must be repressed in such locations to allow sesamoid bone formation. Alternatively, sesamoid bone may be specified prior to the expression of ADAMTS7 and ADAMTS12, a possibility supported by the late onset of Adamts 7 and Adamts12 expression and continued expression into adulthood, so that adult onset, rather than developmental anomalies are seen.

An interesting aspect of $\mathrm{HO}$ in both $\mathrm{Adamts}^{-/-} \mathrm{Adamts} 12^{-1-}$ and $\mathrm{Bgn}^{-10} \mathrm{Fmod}^{-1-}$ mice is its exclusive occurrence in the hindlimbs. Whereas wild-type C57BL/6 mice develop age-related hindlimb $\mathrm{HO}$ as seen here (48), similar changes in the forelimb were not detected and correspondingly little Adamts7 and Adamts12 is expressed in forelimb tendons. This suggests that innate protective mechanisms in hindlimbs may have arisen from evolutionary pressure to counter increased hindlimb susceptibility to $\mathrm{HO}$, such as via increased loading or microtrauma. Indeed, $\mathrm{HO}$ in humans is also more prevalent in hindlimb tendons such as the Achilles tendon following surgery or trauma (49). Nevertheless, reduced ADAMTS7 and ADAMTS12 expression in resected human biceps tendons showed an association with degenerative changes, and in some instances, cartilage metaplasia and HO. Mineralization and ossification, especially within the Achilles tendon, is commonly seen in chronic injury or degeneration, and $\mathrm{HO}$ is a frequent occurrence after repair of ruptured Achilles tendon (48-50). Achilles tendon resections are less readily available than biceps tendons; the findings suggest investigation of ADAMTS7 and ADAMTS12 in Achilles tendons as a major future direction.

Genetically determined $\mathrm{HO}$ in humans typically affects other tissues and different sites. Fibrodysplasia ossificans progressive (FOP), which is caused by ACVR1 mutations, leads to progressive ossification of muscles, tendons, and ligaments (51). Diffuse idiopathic skeletal hyperostosis (Forestier disease) preferentially affects the anterior longitudinal ligament of the spine, and causes enthesophytes of tendons and ligaments (52). Progressive osseous heteroplasia $(\mathrm{POH})$, characterized by $\mathrm{HO}$ extending from dermal and subcutaneous tissues to deep structures in a segmental fashion, is caused by inactivating heterozygous mutation of the $\alpha$ subunit of the G-stimulatory protein of adenylyl cyclase (53). The HO described here in Adamts $7^{-1-}$ Adamts $12^{-1-}$ mice is distinct from these human genetic disorders and also from calcific tendinitis, a painful buildup of calcium in the tendon (54). Could genetic deficiency of either ADAMTS7 or ADAMTS12 cause HO in humans? We predict not, since in humans as in mice, ADAMTS7 and ADAMTS12 are on different chromosomes, such that simultaneous mutation or loss of both copies of both genes is unlikely. Moreover, we show in mice that absence of one leads to compensatory upregulation of the other. Since their coexpression suggests shared regulatory mechanisms, they might both be downregulated coordinately, such as was observed in degenerated human tendons and are likely to be more relevant to acquired tendon disease and surgical repair than genetic tendon anomalies.

In summary, our results suggest that ADAMTS7 and ADAMTS12 are intrinsic tendon components required for maintenance of tenocyte and fibrochondrocyte cell/ECM phenotypes in tendons and menisci. Our studies suggest several possible mechanisms, which may apply concurrently. Collagen fibril anomalies in Adamts $7^{-/}$Adamts12-/- mice are distinct from those seen in other mouse knockouts, and could lead to HO. Additionally, the present data strongly support reduction of the SLRPs biglycan and fibromodulin in Adamts $7^{-1-}$ Adamts12-/- mice as a major potential mechanism of HO. Excess p-SMAD staining was also detected, consistent with a role for SLRPs in regulating TGF- $\beta$ and BMPs and with reduced SLRP content. It is presently not possible to specify if the observed collagen fibril anomalies and $\mathrm{HO}$ in the Adamts $7^{-1-}$ Adamts $12^{-1-}$ mice directly result from loss of proteolytic activity or the PG characteristic of ADAMTS7 and ADAMTS12 defined here. In future studies one could interrogate the role of the GAG modification and catalytic activity individually using CRISPR-Cas9 knockin mutations that produce unmodified ADAMTS7 and ADAMTS12, or catalytically inactive proteases. Identification of the exact sites of GAG attachment shown here expedites this approach. In addition, identification of novel ADAMTS7 and ADAMTS12 substrates is an important future direction to determine how their proteolytic activity might regulate tendon biology.

\section{Methods}

All reagents were from Sigma-Aldrich unless otherwise indicated.

Transgenic mice. Adamts $7^{+/}$mice (Adamts $7^{\text {mla(KOMP)Wtsi) }}$ were obtained from the NIH-supported KOMP Repository (www.komp.org) as a knockout-first allele and were maintained by outbreeding to the C57BL/6 strain. This constitutive null allele has an intragenic $\beta$-gal-neomycin resistance $(\mathrm{NeoR})$ cassette inserted in intron 
4, to provide a reporter for Adamts 7 expression. Because variable lacZ expression was observed, even in Adamts $^{7^{-1}}$ mice, the NeoR cassette was first deleted by intercrossing Adamts $7^{+/-}$mice and Prm1-Cre mice. Progeny of this cross with NeoR deletion were outbred to C57BL/6 wild-type mice to eliminate Prm1-Cre and subsequently maintained in this strain. NeoR deletion gave consistently stronger $\beta$-gal staining and Cre eliminated exons 5 and 6, which encode the Adamts7 propeptide. The Adamts12+/- allele (B6;129S5-Adamts12 ${ }^{\text {tmilex }} / \mathrm{Mmcd}$ ) was generated by Genentech Lexicon by targeting exons 8 through 10 , which encode the catalytic domain, and was recovered from a cryoarchive at the Mutant Mouse Regional Resource Center (University of California-Davis, Davis, CA) (21). Adamts $12^{+/-}$mice were backcrossed 9 generations with C57BL/6 mice. Adamts 7 and Adamts 12 are located on chromosome 9 and 15 respectively, ensuring independent segregation during meiosis. Adamts $7^{-1-}$ Adamts $12^{-1-}$ mice were generated by intercrossing the 2 alleles and subsequently maintained on a C57BL/ 6 background. The offspring of the intercross showed expected Mendelian ratios of adult mice, confirming that the double-null phenotype was not embryonic or perinatal lethal. Whereas only male mice were used for skeletal preparations and measurement of bone length, mice of both sexes were used for x-ray and micro-CT and at embryonic stages (for histology) with no differences between the sexes observed.

Radiographic and micro-CT analysis. Following euthanasia, mouse forelimbs and hindlimbs were dissected and radiographed (DXSPRO In-Vivo; Carestream Health, Inc.). Radiographs were scored by a semiquantitative scoring system (29). Micro-CT analysis was performed on an InSyTe Benchtop CT (Trifoil Imaging Technologies) and 3-dimensional data were processed and analyzed using VolNinja (ImageIQ).

Histology and immunofluorescence microscopy. Mouse forelimbs and hindlimbs were fixed with $4 \%$ paraformaldehyde (PFA) in PBS at $4^{\circ} \mathrm{C}$ for 48 hours. Postnatal limbs were decalcified in 5\% EDTA in PBS for 7-10 days with a change of the solution every other day. Human tendons were fixed in $4 \%$ PFA in PBS at $4^{\circ} \mathrm{C}$ for 48 hours. Paraffin sections $(7 \mu \mathrm{m})$ were used for histochemistry (H\&E, Alcian blue, toluidine blue) or for indirect immunofluorescence. The primary antibodies (Supplemental Table 1) were followed by secondary goat anti-mouse or goat anti-rabbit antibody (A11001 or A11008; 1:200; Invitrogen) treatment. Prior to immunofluorescence, citrate antigen retrieval, i.e., immersion of slides in citrate-EDTA buffer (10 $\mathrm{mM}$ citric acid, $2 \mathrm{mM}$ EDTA, $0.05 \% \mathrm{v} / \mathrm{v}$ Tween-20, $\mathrm{pH}$ 6.2) and microwaving 4 times for 1.5 minutes each at $50 \%$ power in a microwave oven with 30 -second intervals between heating cycles, or chondroitinase $\mathrm{ABC}$ treatment (0.2 U/ml in PBS; C3667, Sigma-Aldrich) was used for anti-ADAMTS7 and ADAMTS12 for 1 hour at $37^{\circ} \mathrm{C}$. Images were obtained using an Olympus BX51 microscope with Leica DFC 7000T camera and Leica Application Suite V4.6 software. Alizarin red-Alcian blue-stained skeleton preparations were performed as previously described (55). Briefly, skinned, eviscerated mice were fixed in $80 \%$ ethanol for 24 hours, dehydrated in 95\% ethanol for 24 hours and acetone for 48 hours, stained in solution (0.1\% Alizarin red S, $0.3 \%$ Alcian blue, $1 \%$ glacial acetic acid in 95\% ethanol) for 48 hours, cleared in $95 \%$ ethanol for 1 hour, muscle tissue gently removed with forceps, and then cleared in a series of increasing $(20 \%-80 \%)$ glycerin $/ 1 \% \mathrm{KOH}$ ratio until storage in $100 \%$ glycerin and photographed. Human tendons were embedded in paraffin and sections were used for $\mathrm{H} \& \mathrm{E}$, toluidine blue, and Masson trichrome staining.

$\beta$-Gal staining and RNA ISH. Tissue from Adamts $7^{+/-}$mice was fixed and stained for $\beta$-gal activity as previously described (56). Adamts12 ISH was performed using RNAScope (Advanced Cell Diagnostics) following the manufacturer's protocol. Briefly, 6- $\mu \mathrm{m}$ sections were deparaffinized and hybridized to a mouse Adamts12 probe set (400531, Advanced Cell Diagnostics) using a HybEZ oven (Advanced Cell Diagnostics) and the RNAScope 2.5 HD Detection Reagent Kit (322360, Advanced Cell Diagnostics).

Cell culture. C3H/10T1/2, clone 8 cells (ATCC, CCL-226) were cultured in 100-mm plastic culture dishes in Dulbecco's Modified Eagle Medium (DMEM) supplemented with 10\% fetal bovine serum (FBS), $100 \mathrm{U} / \mathrm{ml}$ penicillin, and $100 \mu \mathrm{g} / \mathrm{ml}$ streptomycin at $37^{\circ} \mathrm{C}$ in a $5 \% \mathrm{CO}_{2}$ humidified chamber. The cells were transiently transfected with Adamts7 (12) or ADAMTS12 expression plasmids (gift from S. Cal, University of Oviedo, Oviedo, Spain) using a Lipofectamine 3000 Transfection Kit (L3000, Invitrogen). Twenty-four hours after transfection, the cells were detached with trypsin-EDTA and used at $5 \times 10^{5} \mathrm{cells} / \mathrm{ml}$ (for pellet cultures) or $1 \times 10^{7} \mathrm{cells} / \mathrm{ml}$ (for micromass cultures). Pellet cultures were performed as previously described (57). Briefly, $5 \times 10^{5}$ cells were centrifuged at $500 \mathrm{~g}$ for 5 minutes in $15-\mathrm{ml}$ tubes and cultured in chondrogenic medium $\left(10^{-7} \mathrm{M}\right.$ dexamethasone, $0.2 \mathrm{mM}$ L-ascorbic acid-2 phosphate, ITS liquid media supplement, $10 \mathrm{ng} / \mathrm{ml}$ TGF- $\beta 1$ in serum-free DMEM), with a medium change every 48 hours. Micromass cultures were generated as previously described (58). Briefly, $10 \mu 1$ of a $1 \times 10^{7}$ cells $/ \mathrm{ml}$ aliquot was placed in the center of a 24-well dish and the cells were allowed to adhere for 1 hour, after which $1 \mathrm{ml}$ of chondrogenic medium was added and changed every 48 hours. 
TEM and collagen fibril diameter analyses. Achilles tendons dissected from 1-month-old Adamts $7^{-1-}$ Adamts12-1- mice and C57BL/6 wild-type controls were processed for TEM as previously described (59). Briefly, the hindlimbs were fixed in modified Karnovsky's fixative (2.5\% glutaraldehyde, $4 \%$ paraformaldehyde, $0.1 \mathrm{M}$ sodium cacodylate, $8 \mathrm{mM}$ calcium chloride, $\mathrm{pH} 7.4$ ) for 4 hours on a shaker at $4^{\circ} \mathrm{C}$ and transferred to transport solution ( $10 \%$ Karnovsky's fixative, $10 \%$ sucrose, in $0.1 \mathrm{M}$ sodium cacodylate, $\mathrm{pH} 7.4$ ). The tendons were rinsed in cacodylate buffer and postfixed for 1 hour with $1 \%$ osmium tetroxide, dehydrated in ethanol followed by $100 \%$ propylene oxide, infiltrated and embedded in EMbed 812, nadic methyl anhydride, dodecenylsuccinic anhydride, and DMP-30 (Electron Microscopy Sciences) and polymerized overnight at $60^{\circ} \mathrm{C}$. A Leica ultramicrotome was used to prepare thin $(\sim 90 \mathrm{~nm})$ cross-sections, poststained with $2 \%$ aqueous uranyl acetate and $1 \%$ phosphotungstic acid, $\mathrm{pH} 3.2$, and examined and imaged at $80 \mathrm{kV}$ using a JEOL 1400 transmission electron microscope equipped with a Gatan Orius widefield side mount CC Digital camera (Gatan Inc.). Micrographs from nonoverlapping regions of the central portion of the Achilles tendon of control and Adamts $7^{-1-}$ Adamts12-1- mice $(n=3)$ were taken at $\times 60,000$ magnification. A region of interest (ROI) consisted of a minimum of 80 fibrils that were measured using MetaMorph Premier for Olympus, Meta Series 7.65 (Olympus America Inc. and Molecular Devices Inc.). Fibril diameters were measured along the minor axis of the fibril cross-section. Fibril density was obtained as the fibril number per unit area.

Site-directed mutagenesis, transient transfection, deglycosylation, and Western blotting. A plasmid encoding human ADAMTS12 with a C-terminal FLAG tag was used for site-directed mutagenesis (QuikChange Site-Directed Mutagenesis kit, Stratagene) to introduce substitutions at putative GAG attachment sites $\left(S^{1157}\right.$ to Ala, Ser ${ }^{1157}+$ Ser $^{1159}$ to $\left.\mathrm{Ala}^{1157}+\mathrm{Ala}^{1159}\right)$. Plasmids were transfected into HEK293F cells using Fugene 6 (E2691, Promega) and conditioned medium was collected 48-72 hours later. Aliquots of medium were deglycosylated prior to analysis by reducing SDS-PAGE and Western blotting with anti-FLAG M2 antibody (1:1,000; F3165; Sigma-Aldrich). Chondroitinase ABC (C3667, Sigma-Aldrich), keratanase I + II (Seikagaku Corporation), or heparinase I + III (H3917, Sigma-Aldrich) was used for deglycosylation as previously described (12). CS stubs remaining on the ADAMTS12 core protein after chondroitinase ABC digestion were detected by mouse anti-chondroitin 6-sulfate mAb 2035 (EMD Millipore). Mouse tendons were homogenized (Ultra Turrax T2, IKA-Works) in lysis buffer $(0.1 \%$ NP-40, 0.01\% SDS, and $0.05 \%$ sodium deoxycholate in PBS). Extracts were analyzed by $10 \%$ reducing SDS-PAGE. For PG analysis, chondroitinase $\mathrm{ABC}$ digestion for 24 hours at $37^{\circ} \mathrm{C}$ was used prior to electrophoresis. Proteins were electroblotted to polyvinylidene fluoride membranes (IPFL00010, EMD Millipore), incubated with primary antibodies overnight at $4^{\circ} \mathrm{C}$ (Supplemental Table 1), followed by fluorescent secondary antibody goat antimouse or -rabbit (827-08365, 926-68170; 1:5,000; Li-COR) for 1 hour at room temperature. Antibody binding was visualized using an ODYSSEY CLx infrared imaging system (LI-COR Biosciences).

$R N A$ isolation and $q R T-P C R$. Human tendons were snap-frozen and stored at $-80^{\circ} \mathrm{C}$ until use. Following tendon homogenization (Tissue Tearer, Thermo Fisher Scientific) in TRIzol (15596018, Invitrogen) total RNA was extracted from mouse Achilles, quadriceps, and patellar tendon following the manufacturer's instructions. cDNA was generated from $1 \mu \mathrm{g}$ RNA using a High-Capacity cDNA reverse transcription kit following the manufacturer's instructions (4368814, Applied Biosystems). PCR products were electrophoresed in 2\% agarose gels (Gel Logic 100 Imaging System, Kodak). Quantitative RT-PCR (qRT-PCR) was performed using Bullseye EvaGreen qPCR MasterMix (BEQPCR-S, MIDSCI) using an Applied Biosystems 7500 instrument. Three independent PCR amplifications were used for each sample. Gapdh was used as a control for mRNA quantity. Human and mouse primer sequences for RT-PCR and qRT-PCR are in Supplemental Table 2. The $\Delta \Delta \mathrm{Ct}$ method was used to calculate relative mRNA expression levels.

Statistics. Representative data of 3 independent experiments are reported unless otherwise indicated. The 2-tailed, unpaired Student's $t$ test was used to obtain $P$ values. Asterisks indicate differences with statistical significance as follows: ${ }^{*} P \leq 0.01,{ }^{\#} P \leq 0.05$.

Study approval. All mouse experiments were approved by the Cleveland Clinic Institutional Animal Care and Use Committee (protocol 2016-1665). Tendons of the long head of biceps brachii were obtained as tissue discarded at reconstructive shoulder surgery at the Cleveland Clinic under an IRB exemption for use of discarded tissue without patient information (exemption no. 118).

\section{Author contributions}

TJM, DRM, JCH, YD, and SMA conducted experiments and acquired and analyzed the data. TJM, DRM, DEB, and SSA designed the study. TJM and SSA wrote the manuscript. 


\section{Acknowledgments}

The authors would like to acknowledge Elisabeth Cahill, Rick Rozic, and Ronald Midura for technical assistance and discussions, and surgeons and patients of the Department of Orthopaedic Surgery at the Cleveland Clinic for discarded tendon tissue. Funding was provided by the NIH (Program of Excellence in Glycoscience award HL107147 to S.S. Apte and F32AR063548 to T.J. Mead) and the David and Lindsay Morgenthaler Postdoctoral Fellowship (to T.J. Mead). S.S. Apte is supported by an American Heart Association-Paul G. Allen Frontiers Group Distinguished Investigator Award.

Address correspondence to: Suneel S. Apte, Department of Biomedical Engineering-ND20, Lerner Research Institute, Cleveland Clinic, 9500 Euclid Avenue, Cleveland, Ohio 44195, USA. Phone: 216.445.3278; E-mail: aptes@ccf.org.

1. Hubmacher D, Apte SS. ADAMTS proteins as modulators of microfibril formation and function. Matrix Biol. 2015;47:34-43.

2. Lu P, Takai K, Weaver VM, Werb Z. Extracellular matrix degradation and remodeling in development and disease. Cold Spring Harb Perspect Biol. 2011;3(12): a005058.

3. Prox J, Arnold P, Becker-Pauly C. Meprin $\alpha$ and meprin $\beta$ : Procollagen proteinases in health and disease. Matrix Biol. 2015;4446:7-13.

4. Apte SS. A disintegrin-like and metalloprotease (reprolysin-type) with thrombospondin type 1 motif (ADAMTS) superfamily: functions and mechanisms. J Biol Chem. 2009;284(46):31493-31497.

5. Dubail J, Apte SS. Insights on ADAMTS proteases and ADAMTS-like proteins from mammalian genetics. Matrix Biol. 2015;44-46:24-37.

6. Colige A, et al. Human Ehlers-Danlos syndrome type VII C and bovine dermatosparaxis are caused by mutations in the procollagen I N-proteinase gene. Am J Hum Genet. 1999;65(2):308-317.

7. Bayliss MT, Hutton S, Hayward J, Maciewicz RA. Distribution of aggrecanase (ADAMts 4/5) cleavage products in normal and osteoarthritic human articular cartilage: the influence of age, topography and zone of tissue. Osteoarthr Cartil. 2001;9(6):553-560.

8. Malfait AM, Liu RQ, Ijiri K, Komiya S, Tortorella MD. Inhibition of ADAM-TS4 and ADAM-TS5 prevents aggrecan degradation in osteoarthritic cartilage. J Biol Chem. 2002;277(25):22201-22208.

9. Song RH, et al. Aggrecan degradation in human articular cartilage explants is mediated by both ADAMTS-4 and ADAMTS-5. Arthritis Rheum. 2007;56(2):575-585.

10. Le Goff C, Cormier-Daire V. The ADAMTS(L) family and human genetic disorders. Hum Mol Genet. 2011;20(R2):R163-R167.

11. Huxley-Jones J, Apte SS, Robertson DL, Boot-Handford RP. The characterisation of six ADAMTS proteases in the basal chordate Ciona intestinalis provides new insights into the vertebrate ADAMTS family. Int J Biochem Cell Biol. 2005;37(9):1838-1845

12. Somerville RP, et al. ADAMTS7B, the full-length product of the ADAMTS7 gene, is a chondroitin sulfate proteoglycan containing a mucin domain. J Biol Chem. 2004;279(34):35159-35175.

13. Reilly MP, et al. Identification of ADAMTS7 as a novel locus for coronary atherosclerosis and association of ABO with myocardial infarction in the presence of coronary atherosclerosis: two genome-wide association studies. Lancet. 2011;377(9763):383-392.

14. Bauer RC, et al. Knockout of Adamts7, a novel coronary artery disease locus in humans, reduces atherosclerosis in mice. Circulation. 2015;131(13):1202-1213.

15. Pu X, et al. ADAMTS7 cleavage and vascular smooth muscle cell migration is affected by a coronary-artery-disease-associated variant. Am J Hum Genet. 2013;92(3):366-374.

16. Kessler T, et al. ADAMTS-7 inhibits re-endothelialization of injured arteries and promotes vascular remodeling through cleavage of thrombospondin-1. Circulation. 2015;131(13):1191-1201.

17. Liu CJ, et al. ADAMTS-7: a metalloproteinase that directly binds to and degrades cartilage oligomeric matrix protein. FASEB J. 2006;20(7):988-990.

18. Liu CJ, et al. ADAMTS-12 associates with and degrades cartilage oligomeric matrix protein. J Biol Chem. 2006;281(23):15800-15808.

19. Liu CJ. The role of ADAMTS-7 and ADAMTS-12 in the pathogenesis of arthritis. Nat Clin Pract Rheumatol. 2009;5(1):38-45.

20. Zhang Q, Huang M, Wang X, Xu X, Ni M, Wang Y. Negative effects of ADAMTS-7 and ADAMTS-12 on endplate cartilage differentiation. J Orthop Res. 2012;30(8):1238-1243.

21. El Hour M, et al. Higher sensitivity of Adamts12-deficient mice to tumor growth and angiogenesis. Oncogene. 2010;29(20):3025-3032

22. Ezura Y, Chakravarti S, Oldberg A, Chervoneva I, Birk DE. Differential expression of lumican and fibromodulin regulate collagen fibrillogenesis in developing mouse tendons. J Cell Biol. 2000;151(4):779-788.

23. Zhang G, et al. Decorin regulates assembly of collagen fibrils and acquisition of biomechanical properties during tendon development. J Cell Biochem. 2006;98(6):1436-1449.

24. Zhang G, et al. Development of tendon structure and function: regulation of collagen fibrillogenesis. J Musculoskelet Neuronal Interact. 2005;5(1):5-21.

25. Nandadasa S, Foulcer S, Apte SS. The multiple, complex roles of versican and its proteolytic turnover by ADAMTS proteases during embryogenesis. Matrix Biol. 2014;35:34-41.

26. Brinkmann T, Weilke C, Kleesiek K. Recognition of acceptor proteins by UDP-D-xylose proteoglycan core protein beta-Dxylosyltransferase. J Biol Chem. 1997;272(17):11171-11175.

27. Chen S, Birk DE. The regulatory roles of small leucine-rich proteoglycans in extracellular matrix assembly. FEBS J. 2013;280(10):2120-2137.

28. Kalamajski S, Oldberg A. The role of small leucine-rich proteoglycans in collagen fibrillogenesis. Matrix Biol. 2010;29(4):248-253. 
29. Ameye L, Aria D, Jepsen K, Oldberg A, Xu T, Young MF. Abnormal collagen fibrils in tendons of biglycan/fibromodulindeficient mice lead to gait impairment, ectopic ossification, and osteoarthritis. FASEB J. 2002;16(7):673-680.

30. Reznikoff CA, Brankow DW, Heidelberger C. Establishment and characterization of a cloned line of C3H mouse embryo cells sensitive to postconfluence inhibition of division. Cancer Res. 1973;33(12):3231-3238.

31. Denker AE, Haas AR, Nicoll SB, Tuan RS. Chondrogenic differentiation of murine C3H10T1/2 multipotential mesenchymal cells: I. Stimulation by bone morphogenetic protein-2 in high-density micromass cultures. Differentiation. 1999;64(2):67-76.

32. Denker AE, Nicoll SB, Tuan RS. Formation of cartilage-like spheroids by micromass cultures of murine C3H10T1/2 cells upon treatment with transforming growth factor-beta 1. Differentiation. 1995;59(1):25-34.

33. Thelin MA, et al. Biological functions of iduronic acid in chondroitin/dermatan sulfate. FEBS J. 2013;280(10):2431-2446

34. Hascall VC, Sandy JD, Handley CJ. In: Archer CW, Caterson B, Benjamin M, Ralphs JR, eds. Biology of the Synovial Joint. Amsterdam, the Netherlands: Harwood Academic Publishers; 1999:101-120. ISBN 90-5702-327-X.

35. Le Jan S, et al. Functional overlap between chondroitin and heparan sulfate proteoglycans during VEGF-induced sprouting angiogenesis. Arterioscler Thromb Vasc Biol. 2012;32(5):1255-1263.

36. Whalen DM, Malinauskas T, Gilbert RJ, Siebold C. Structural insights into proteoglycan-shaped Hedgehog signaling. Proc Natl Acad Sci USA. 2013;110(41):16420-16425.

37. McCulloch DR, et al. ADAMTS metalloproteases generate active versican fragments that regulate interdigital web regression Dev Cell. 2009;17(5):687-698.

38. Enomoto H, et al. Cooperation of two ADAMTS metalloproteases in closure of the mouse palate identifies a requirement for versican proteolysis in regulating palatal mesenchyme proliferation. Development. 2010;137(23):4029-4038.

39. Bai XH, et al. ADAMTS-7, a direct target of PTHrP, adversely regulates endochondral bone growth by associating with and inactivating GEP growth factor. Mol Cell Biol. 2009;29(15):4201-4219.

40. Bai XH, Wang DW, Luan Y, Yu XP, Liu CJ. Regulation of chondrocyte differentiation by ADAMTS-12 metalloproteinase depends on its enzymatic activity. Cell Mol Life Sci. 2009;66(4):667-680.

41. Bi Y, et al. Identification of tendon stem/progenitor cells and the role of the extracellular matrix in their niche. Nat Med. 2007;13(10):1219-1227.

42. Kilts T, et al. Potential roles for the small leucine-rich proteoglycans biglycan and fibromodulin in ectopic ossification of tendon induced by exercise and in modulating rotarod performance. Scand J Med Sci Sports. 2009;19(4):536-546.

43. Wang L, et al. Fibromodulin and biglycan modulate periodontium through TGFß/BMP signaling. J Dent Res. 2014;93(8):780-787.

44. Schweitzer R, et al. Analysis of the tendon cell fate using Scleraxis, a specific marker for tendons and ligaments. Development. 2001;128(19):3855-3866.

45. Zhang $\mathrm{K}$, et al. Tendon mineralization is progressive and associated with deterioration of tendon biomechanical properties, and requires BMP-Smad signaling in the mouse Achilles tendon injury model. Matrix Biol. 2016;52-54:315-324.

46. Suzuki H, et al. Gene targeting of the transcription factor Mohawk in rats causes heterotopic ossification of Achilles tendon via failed tenogenesis. Proc Natl Acad Sci USA. 2016;113(28):7840-7845.

47. Sarin VK, Erickson GM, Giori NJ, Bergman AG, Carter DR. Coincident development of sesamoid bones and clues to their evolution. Anat Rec. 1999;257(5):174-180.

48. O'Brien EJ, Frank CB, Shrive NG, Hallgrímsson B, Hart DA. Heterotopic mineralization (ossification or calcification) in tendinopathy or following surgical tendon trauma. Int J Exp Pathol. 2012;93(5):319-331.

49. Richards PJ, Braid JC, Carmont MR, Maffulli N. Achilles tendon ossification: pathology, imaging and aetiology. Disabil Rehabil. 2008;30(20-22):1651-1665.

50. Järvinen M, Józsa L, Kannus P, Järvinen TL, Kvist M, Leadbetter W. Histopathological findings in chronic tendon disorders. Scand J Med Sci Sports. 1997;7(2):86-95.

51. Shore EM, et al. A recurrent mutation in the BMP type I receptor ACVR1 causes inherited and sporadic fibrodysplasia ossificans progressiva. Nat Genet. 2006;38(5):525-527.

52. Childs SG. Diffuse idiopathic skeletal hyperostosis: Forestier's disease. Orthop Nurs. 2004;23(6):375-82; quiz 383.

53. Kaplan FS, Shore EM. Progressive osseous heteroplasia. J Bone Miner Res. 2000;15(11):2084-2094.

54. ElShewy MT. Calcific tendinitis of the rotator cuff. World J Orthop. 2016;7(1):55-60.

55. Mead TJ, Yutzey KE. Notch pathway regulation of chondrocyte differentiation and proliferation during appendicular and axial skeleton development. Proc Natl Acad Sci USA. 2009;106(34):14420-14425.

56. McCulloch DR, Le Goff C, Bhatt S, Dixon LJ, Sandy JD, Apte SS. Adamts5, the gene encoding a proteoglycan-degrading metalloprotease, is expressed by specific cell lineages during mouse embryonic development and in adult tissues. Gene Expr Patterns. 2009;9(5):314-323.

57. Zhao L, Li G, Chan KM, Wang Y, Tang PF. Comparison of multipotent differentiation potentials of murine primary bone marrow stromal cells and mesenchymal stem cell line C3H10T1/2. Calcif Tissue Int. 2009;84(1):56-64.

58. Haas AR, Tuan RS. Murine C3H10T1/2 multipotential cells as an in vitro model of mesenchymal chondrogenesis. Methods Mol Biol. 2000;137:383-389.

59. Dunkman AA, et al. Decorin expression is important for age-related changes in tendon structure and mechanical properties Matrix Biol. 2013;32(1):3-13. 